\title{
Mann-Type Extragradient Methods for General Systems of Variational Inequalities with Multivalued Variational Inclusion Constraints in Banach Spaces
}

\author{
Lu-Chuan Ceng, ${ }^{1}$ Abdul Latif, ${ }^{2}$ and Saleh A. Al-Mezel ${ }^{2}$ \\ ${ }^{1}$ Department of Mathematics, Shanghai Normal University, and Scientific Computing Key Laboratory of Shanghai Universities, \\ Shanghai 200234, China \\ ${ }^{2}$ Department of Mathematics, King Abdulaziz University, P.O. Box 80203, Jeddah 21589, Saudi Arabia
}

Correspondence should be addressed to Abdul Latif; alatif@kau.edu.sa

Received 13 September 2013; Accepted 30 October 2013

Academic Editor: Jen-Chih Yao

Copyright (C) $2013 \mathrm{Lu}$-Chuan Ceng et al. This is an open access article distributed under the Creative Commons Attribution License, which permits unrestricted use, distribution, and reproduction in any medium, provided the original work is properly cited.

\begin{abstract}
We introduce Mann-type extragradient methods for a general system of variational inequalities with solutions of a multivalued variational inclusion and common fixed points of a countable family of nonexpansive mappings in real smooth Banach spaces. Here the Mann-type extragradient methods are based on Korpelevich's extragradient method and Mann iteration method. We first consider and analyze a Mann-type extragradient algorithm in the setting of uniformly convex and 2-uniformly smooth Banach space and then another Mann-type extragradient algorithm in a smooth and uniformly convex Banach space. Under suitable assumptions, we derive some weak and strong convergence theorems. The results presented in this paper improve, extend, supplement, and develop the corresponding results announced in the earlier and very recent literature.
\end{abstract}

\section{Introduction}

Let $X$ be a real Banach space whose dual space is denoted by $X^{*}$. The normalized duality mapping $J: X \rightarrow 2^{X^{*}}$ is defined by

$$
J(x)=\left\{x^{*} \in X^{*}:\left\langle x, x^{*}\right\rangle=\|x\|^{2}=\left\|x^{*}\right\|^{2}\right\}, \quad \forall x \in X,
$$

where $\langle\cdot, \cdot\rangle$ denotes the generalized duality pairing. It is an immediate consequence of the Hahn-Banach theorem that $J(x)$ is nonempty for each $x \in X$. Let $C$ be a nonempty closed convex subset of $X$. A mapping $T: C \rightarrow C$ is called nonexpansive if $\|T x-T y\| \leq\|x-y\|$ for every $x, y \in C$. The set of fixed points of $T$ is denoted by $\operatorname{Fix}(T)$. We use the notation $\rightarrow$ to indicate the weak convergence and the one $\rightarrow$ to indicate the strong convergence. A mapping $A: C \rightarrow X$ is said to be as follows:

(i) accretive if for each $x, y \in C$ there exists $j(x-y) \in$ $J(x-y)$ such that

$$
\langle A x-A y, j(x-y)\rangle \geq 0
$$

(ii) $\alpha$-strongly accretive if for each $x, y \in C$ there exists $j(x-y) \in J(x-y)$ such that

$$
\langle A x-A y, j(x-y)\rangle \geq \alpha\|x-y\|^{2},
$$

for some $\alpha \in(0,1)$;

(iii) $\beta$-inverse-strongly-accretive if for each $x, y \in C$ there exists $j(x-y) \in J(x-y)$ such that

$$
\langle A x-A y, j(x-y)\rangle \geq \beta\|A x-A y\|^{2},
$$

for some $\beta>0$;

(iv) $\lambda$-strictly pseudocontractive if for each $x, y \in C$ there exists $j(x-y) \in J(x-y)$ such that

$$
\langle A x-A y, j(x-y)\rangle \leq\|x-y\|^{2}-\lambda\|x-y-(A x-A y)\|^{2},
$$

for some $\lambda \in(0,1)$. 
Let $U=\{x \in X:\|x\|=1\}$ denote the unite sphere of $X$. A Banach space $X$ is said to be uniformly convex if for each $\epsilon \in(0,2]$, there exists $\delta>0$ such that for all $x, y \in U$

$$
\|x-y\| \geq \epsilon \Longrightarrow \frac{\|x+y\|}{2} \leq 1-\delta .
$$

It is known that a uniformly convex Banach space is reflexive and strictly convex. A Banach space $X$ is said to be smooth if the limit

$$
\lim _{t \rightarrow 0} \frac{\|x+t y\|-\|x\|}{t}
$$

exists for all $x, y \in U$; in this case, $X$ is also said to have a Gáteaux differentiable norm. Moreover, it is said to be uniformly smooth if this limit is attained uniformly for $x, y \in$ $U$. The norm of $X$ is said to be the Fréchet differential if for each $x \in U$, this limit is attained uniformly for $y \in U$. In the meantime, we define a function $\rho:[0, \infty) \rightarrow[0, \infty)$ called the modulus of smoothness of $X$ as follows:

$$
\begin{array}{r}
\rho(\tau)=\sup \left\{\frac{1}{2}(\|x+y\|+\|x-y\|)-1:\right. \\
x, y \in X,\|x\|=1,\|y\|=\tau\} .
\end{array}
$$

It is known that $X$ is uniformly smooth if and only if $\lim _{\tau \rightarrow 0} \rho(\tau) / \tau=0$. Let $q$ be a fixed real number with $1<q \leq$ 2 . Then a Banach space $X$ is said to be $q$-uniformly smooth if there exists a constant $c>0$ such that $\rho(\tau) \leq c \tau^{q}$ for all $\tau>0$. As pointed out in [1], no Banach space is $q$-uniformly smooth for $q>2$. In addition, it is also known that $J$ is single-valued if and only if $X$ is smooth, whereas if $X$ is uniformly smooth, then $J$ is norm-to-norm uniformly continuous on bounded subsets of $X$.

Very recently, Cai and $\mathrm{Bu}$ [2] considered the following general system of variational inequalities (GSVI) in a real smooth Banach space $X$, which involves finding $\left(x^{*}, y^{*}\right) \in$ $C \times C$ such that

$$
\begin{aligned}
& \left\langle\mu_{1} B_{1} y^{*}+x^{*}-y^{*}, J\left(x-x^{*}\right)\right\rangle \geq 0, \quad \forall x \in C, \\
& \left\langle\mu_{2} B_{2} x^{*}+y^{*}-x^{*}, J\left(x-y^{*}\right)\right\rangle \geq 0, \quad \forall x \in C,
\end{aligned}
$$

where $C$ is a nonempty, closed, and convex subset of $X$; $B_{1}, B_{2}: C \rightarrow X$ are two nonlinear mappings, and $\mu_{1}$ and $\mu_{2}$ are two positive constants. Here the set of solutions of GSVI (9) is denoted by $\operatorname{GSVI}\left(C, B_{1}, B_{2}\right)$. In particular, if $X=H$, a real Hilbert space, then GSVI (9) reduces to the following GSVI of finding $\left(x^{*}, y^{*}\right) \in C \times C$ such that

$$
\begin{array}{ll}
\left\langle\mu_{1} B_{1} y^{*}+x^{*}-y^{*}, x-x^{*}\right\rangle \geq 0, & \forall x \in C, \\
\left\langle\mu_{2} B_{2} x^{*}+y^{*}-x^{*}, x-y^{*}\right\rangle \geq 0, & \forall x \in C,
\end{array}
$$

where $\mu_{1}$ and $\mu_{2}$ are two positive constants. The set of solutions of problem (10) is still denoted by $\operatorname{GSVI}\left(C, B_{1}, B_{2}\right)$.

Recently, Ceng et al. [3] transformed problem (10) into a fixed point problem in the following way.
Lemma 1 (see [3]). For given $\bar{x}, \bar{y} \in C,(\bar{x}, \bar{y})$ is a solution of problem (10) if and only if $\bar{x}$ is a fixed point of the mapping $G: C \rightarrow C$ defined by

$$
\begin{array}{r}
G(x)=P_{C}\left[P_{C}\left(x-\mu_{2} B_{2} x\right)-\mu_{1} B_{1} P_{C}\left(x-\mu_{2} B_{2} x\right)\right], \\
\forall x \in C,
\end{array}
$$

where $\bar{y}=P_{C}\left(\bar{x}-\mu_{2} B_{2} \bar{x}\right)$ and $P_{C}$ is the the projection of $H$ onto C.

In particular, if the mappings $B_{i}: C \rightarrow H$ is $\beta_{i}$-inverse strongly monotone for $i=1,2$, then the mapping $G$ is nonexpansive provided $\mu_{i} \in\left(0,2 \beta_{i}\right)$ for $i=1,2$.

Define the mapping $G: C \rightarrow C$ as follows:

$$
G(x):=\Pi_{C}\left(I-\mu_{1} B_{1}\right) \Pi_{C}\left(I-\mu_{2} B_{2}\right) x, \quad \forall x \in C .
$$

The fixed point set of $G$ is denoted by $\Omega$.

Let $C B(X)$ be the family of all nonempty, closed and bounded subsets of a real smooth Banach space $X$. Also, we denote by $H(\cdot, \cdot)$ the Hausdorff metric on $C B(X)$ defined by

$$
\begin{array}{r}
H(A, B):=\max \left\{\sup _{x \in B} \inf _{y \in A} d(x, y), \sup _{x \in A} \inf _{y \in B} d(x, y)\right\}, \\
\forall A, B \in C B(X) .
\end{array}
$$

Let $T$ and $F: X \rightarrow C B(X)$ be two multivalued mappings, let $A: D(A) \subset X \rightarrow 2^{X}$ be an $m$-accretive mapping, let $g: X \rightarrow D(A)$ be a single-valued mapping, and let $N(\cdot, \cdot)$ : $X \times X \rightarrow X$ be a nonlinear mapping. Then for any given $v \in X, \lambda>0$, Chidume et al. [4] introduced and studied the multivalued variational inclusion (MVVI) of finding $x \in$ $D(A)$ such that $(x, w, k)$ is a solution of the following:

$$
v \in N(w, k)+\lambda A(g(x)), \quad \forall w \in T x, k \in F x .
$$

If $v=0$ and $\lambda=1$, then the MVVI (14) reduces to the problem of finding $x \in D(A)$ such that $(x, w, k)$ is a solution of the following:

$$
0 \in N(w, k)+A(g(x)), \quad \forall w \in T x, k \in F x .
$$

We denote by $\Gamma$ the set of such solutions $x$ for MVVI (15).

The authors [4] first established an existence theorem for MVVI (14) in smooth Banach space $X$ and then proved that the sequence generated by their iterative algorithm converges strongly to a solution of MVVI (15).

Theorem 2 (see [4, Theorem 3.2]). Let $X$ be a real smooth Banach space. Let $T$ and $F: X \rightarrow C B(X)$, let $A: D(A) \subset$ $X \rightarrow 2^{X}$ be three multivalued mappings, let $g: X \rightarrow D(A)$ be a single-valued mapping, and let $N(\cdot, \cdot): X \times X \rightarrow X$ be a single-valued continuous mapping satisfying the following conditions:

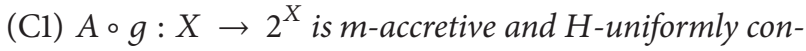
tinuous;

(C2) $T: X \rightarrow C B(X)$ is H-uniformly continuous;

(C3) $F: X \rightarrow C B(X)$ is H-uniformly continuous; 
(C4) the mapping $x \mapsto N(x, y)$ is $\phi$-strongly accretive and $\mu$-H-Lipschitz with respect to the mapping $T$, where $\phi$ : $[0, \infty) \rightarrow[0, \infty)$ is a strictly increasing function with $\phi(0)=0$;

(C5) the mapping $y \mapsto N(x, y)$ is accretive and $\xi-H$ Lipschitz with respect to the mapping $F$.

For arbitrary $x_{0} \in D(A)$ define the sequence $\left\{x_{n}\right\}$ iteratively by

$$
x_{n+1}=x_{n}-\sigma_{n}\left(N\left(w_{n}, k_{n}\right)+u_{n}\right), \quad u_{n} \in A\left(g\left(x_{n}\right)\right) \text {, }
$$

where $\left\{u_{n}\right\}$ is defined by

$$
\begin{array}{r}
\left\|u_{n}-u_{n+1}\right\| \leq(1+\varepsilon) H\left(A\left(g\left(x_{n+1}\right)\right), A\left(g\left(x_{n}\right)\right)\right), \\
\forall n \geq 0,
\end{array}
$$

for any $w_{n} \in T x_{n}, k_{n} \in F x_{n}$ and some $\varepsilon>0$, where $\left\{\sigma_{n}\right\}$ is a positive real sequence such that $\lim _{n \rightarrow \infty} \sigma_{n}=0$ and $\sum_{n=0}^{\infty} \sigma_{n}=$ $\infty$. Then, there exists $\bar{d}>0$ such that for $0<\sigma_{n} \leq \bar{d}$, for all $n \geq 0,\left\{x_{n}\right\}$ converges strongly to $\bar{x} \in \Gamma$; and for any $w \in T \bar{x}$, $k \in F \bar{x},(\bar{x}, w, k)$ is a solution of the MVVI (15).

Let $C$ be a nonempty closed convex subset of a real smooth Banach space $X$ and let $\Pi_{C}$ be a sunny nonexpansive retraction from $X$ onto $C$. Motivated and inspired by the research going on this area, we introduce Mann-type extragradient methods for finding solutions of the GSVI (9) which are also ones of the MVVI (15) and common fixed points of a countable family of nonexpansive mappings. Here the Mann-type extragradient methods are based on Korpelevich's extragradient method and Mann iteration method. We first consider and analyze a Mann-type extragradient algorithm in the setting of uniformly convex and 2-uniformly smooth Banach space, and then another Mann-type extragradient algorithm in a smooth and uniformly convex Banach space. Under suitable assumptions, we derive some weak and strong convergence theorems. The results presented in this paper improve, extend, supplement, and develop the corresponding results announced in the earlier and very recent literature; see for example, [2-10].

\section{Preliminaries}

Let $X$ be a real Banach space with dual $X^{*}$. We denote by $J$ the normalized duality mapping from $X$ to $2^{X^{*}}$ defined by

$$
J(x)=\left\{x^{*} \in X^{*}:\left\langle x, x^{*}\right\rangle=\|x\|^{2}=\left\|x^{*}\right\|^{2}\right\},
$$

where $\langle\cdot, \cdot\rangle$ denotes the generalized duality pairing. Throughout this paper the single-valued normalized duality map is still denoted by $J$. Unless otherwise stated, we assume that $X$ is a smooth Banach space with dual $X^{*}$.

A multivalued mapping $A: D(A) \subseteq X \rightarrow 2^{X}$ is said to be as follows:

(i) accretive, if

$$
\langle u-v, J(x-y)\rangle \geq 0, \quad \forall u \in A x, \quad v \in A y ;
$$

(ii) $m$-accretive, if $A$ is accretive and $(I+r A)(D(A))=X$, for all $r>0$, where $I$ is the identity mapping;

(iii) $\zeta$-inverse strongly accretive, if there exists a constant $\zeta>0$ such that

$$
\langle u-v, J(x-y)\rangle \geq \zeta\|u-v\|^{2}, \quad \forall u \in A x, \quad v \in A y
$$

(iv) $\phi$-strongly accretive, if there exists a strictly increasing continuous function $\phi:[0, \infty) \rightarrow[0, \infty)$ with $\phi(0)=0$ such that

$$
\begin{array}{r}
\langle u-v, J(x-y)\rangle \geq \phi(\|x-y\|)\|x-y\|, \\
\forall u \in A x, \quad v \in A y
\end{array}
$$

(v) $\phi$-expansive, if

$$
\|u-v\| \geq \phi(\|x-y\|), \quad \forall u \in A x, v \in A y .
$$

It is easy to see that if $A$ is $\phi$-strongly accretive, then $A$ is $\phi$-expansive.

A mapping $T: X \rightarrow C B(X)$ is said to be $H$-uniformly continuous, if for any given $\varepsilon>0$ there exists a $\delta>0$ such that whenever $\|x-y\|<\delta$ then $H(T x, T y)<\varepsilon$.

A mapping $N: X \times X \rightarrow X$ is $\phi$-strongly accretive, with respect to $T: X \rightarrow C B(X)$, in the first argument if

$$
\begin{aligned}
& \langle N(u, z)-N(v, z), J(x-y)\rangle \\
& \quad \geq \phi(\|x-y\|)\|x-y\|, \quad \forall u \in T x, v \in T y .
\end{aligned}
$$

A mapping $S: X \rightarrow 2^{X}$ is called lower semicontinuous if $S^{-1}(V):=\{x \in X: S x \cap V \neq \emptyset\}$ is open in $X$ whenever $V \subset Y$ is open.

We list some propositions and lemmas that will be used in the sequel.

Proposition 3 (see [11]). Let $\left\{\lambda_{n}\right\}$ and $\left\{b_{n}\right\}$ be sequences of nonnegative numbers and $\left\{\alpha_{n}\right\} \subset(0,1)$ a sequence satisfying the conditions that $\left\{\lambda_{n}\right\}$ is bounded, $\sum_{n=0}^{\infty} \alpha_{n}=\infty$, and $b_{n} \rightarrow$ 0 , as $n \rightarrow \infty$. Let the recursive inequality

$$
\lambda_{n+1}^{2} \leq \lambda_{n}^{2}-2 \alpha_{n} \psi\left(\lambda_{n+1}\right)+2 \alpha_{n} b_{n} \lambda_{n+1}, \quad \forall n \geq 0,
$$

be given where $\psi:[0, \infty) \rightarrow[0, \infty)$ is a strictly increasing function such that it is positive on $(0, \infty)$ and $\psi(0)=0$. Then $\lambda_{n} \rightarrow 0$, as $n \rightarrow \infty$.

Proposition 4 (see [12]). Let $X$ be a real smooth Banach space. Let $T$ and $F: X \rightarrow 2^{X}$ be two multivalued mappings, and let $N(\cdot, \cdot): X \times X \rightarrow X$ be a nonlinear mapping satisfying the following conditions:

(i) the mapping $x \mapsto N(x, y)$ is $\phi$-strongly accretive with respect to the mapping $T$;

(ii) the mapping $y \mapsto N(x, y)$ is accretive with respect to the mapping $F$.

Then the mapping $S: X \rightarrow 2^{X}$ defined by $S x=N(T x, F x)$ is $\phi$-strongly accretive. 
Proposition 5 (see [13]). Let $X$ be a real Banach space and let $S: X \rightarrow 2^{X} \backslash\{\emptyset\}$ be a lower semicontinuous and $\phi$-strongly accretive mapping; then for any $x \in X, S x$ is a one-point set; that is, $S$ is a single-valued mapping.

Recall that a Banach space $X$ is said to satisfy Opial's condition, if whenever $\left\{x_{n}\right\}$ is a sequence in $X$ which converges weakly to $x$ as $n \rightarrow \infty$, then

$$
\begin{array}{r}
\limsup _{n \rightarrow \infty}\left\|x_{n}-x\right\|<\limsup _{n \rightarrow \infty}\left\|x_{n}-y\right\|, \\
\forall y \in X \text { with } x \neq y .
\end{array}
$$

Lemma 6 (Demiclosedness principle; see [14, Lemma 2]). Let $C$ be a nonempty closed convex subset of a reflexive Banach space $X$ that satisfies Opial's condition and suppose that $T$ : $C \rightarrow X$ is nonexpansive. Then the mapping $I-T$ is demiclosed at zero, that is, $x_{n} \rightarrow x$ and $x_{n}-T x_{n} \rightarrow 0$ imply $x=T x$; that is, $x \in \operatorname{Fix}(T)$.

The following lemma is an immediate consequence of the subdifferential inequality of the function $(1 / 2)\|\cdot\|^{2}$.

Lemma 7. In a real smooth Banach space $X$, there holds the inequality

$$
\|x+y\|^{2} \leq\|x\|^{2}+2\langle y, J(x+y)\rangle, \quad \forall x, y \in X .
$$

Let $D$ be a subset of $C$ and let $\Pi$ be a mapping of $C$ into $D$. Then $\Pi$ is said to be sunny if

$$
\Pi[\Pi(x)+t(x-\Pi(x))]=\Pi(x),
$$

whenever $\Pi(x)+t(x-\Pi(x)) \in C$ for $x \in C$ and $t \geq 0$. A mapping $\Pi$ of $C$ into itself is called a retraction if $\Pi^{2}=\Pi$. If a mapping $\Pi$ of $C$ into itself is a retraction, then $\Pi(z)=z$ for every $z \in R(\Pi)$, where $R(\Pi)$ is the range of $\Pi$. A subset $D$ of $C$ is called a sunny nonexpansive retract of $C$ if there exists a sunny nonexpansive retraction from $C$ onto $D$. The following lemma concerns the sunny nonexpansive retraction.

Lemma 8 (see [15]). Let $C$ be a nonempty closed convex subset of a real smooth Banach space $X$. Let $D$ be a nonempty subset of $C$. Let $\Pi$ be a retraction of $C$ onto $D$. Then the following are equivalent:

(i) $\Pi$ is sunny and nonexpansive;

(ii) $\|\Pi(x)-\Pi(y)\|^{2} \leq\langle x-y, J(\Pi(x)-\Pi(y))\rangle$, for all $x, y \in C$;

(iii) $\langle x-\Pi(x), J(y-\Pi(x))\rangle \leq 0$, for all $x \in C, y \in D$.

It is well known that if $X=H$ a Hilbert space, then a sunny nonexpansive retraction $\Pi_{C}$ is coincident with the metric projection from $X$ onto $C$; that is, $\Pi_{C}=P_{C}$. If $C$ is a nonempty closed convex subset of a strictly convex and uniformly smooth Banach space $X$ and if $T: C \rightarrow C$ is a nonexpansive mapping with the fixed point set $\operatorname{Fix}(T) \neq \emptyset$, then the set $\operatorname{Fix}(T)$ is a sunny nonexpansive retract of $C$. The following result is an easy consequence of Lemma 8.
Lemma 9. Let $C$ be a nonempty closed convex subset of a smooth Banach space $X$. Let $\Pi_{C}$ be a sunny nonexpansive retraction from $X$ onto $C$ and let $B_{1}, B_{2}: C \rightarrow X$ be nonlinear mappings. For given $x^{*}, y^{*} \in C,\left(x^{*}, y^{*}\right)$ is a solution of GSVI (9) if and only if $x^{*}=\Pi_{C}\left(y^{*}-\mu_{1} B_{1} y^{*}\right)$, where $y^{*}=\Pi_{C}\left(x^{*}-\right.$ $\left.\mu_{2} B_{2} x^{*}\right)$.

In terms of Lemma 9, we observe that

$$
x^{*}=\Pi_{C}\left[\Pi_{C}\left(x^{*}-\mu_{2} B_{2} x^{*}\right)-\mu_{1} B_{1} \Pi_{C}\left(x^{*}-\mu_{2} B_{2} x^{*}\right)\right],
$$

which implies that $x^{*}$ is a fixed point of the mapping $G$. Throughout this paper, the set of fixed points of the mapping $G$ is denoted by $\Omega$.

Lemma 10 (see [16]). Given a number $r>0$. A real Banach space $X$ is uniformly convex if and only if there exists a continuous strictly increasing function $\varphi:[0, \infty) \rightarrow[0, \infty)$, $\varphi(0)=0$, such that

$$
\begin{aligned}
\|\lambda x+(1-\lambda) y\|^{2} \leq & \lambda\|x\|^{2}+(1-\lambda)\|y\|^{2} \\
& -\lambda(1-\lambda) \varphi(\|x-y\|),
\end{aligned}
$$

for all $\lambda \in[0,1]$, and $x, y \in X$ such that $\|x\| \leq r$ and $\|y\| \leq r$.

Lemma 11 (see [17]). Let $C$ be a nonempty closed convex subset of a Banach space $X$. Let $S_{0}, S_{1}, \ldots$ be a sequence of mappings of $C$ into itself. Suppose that $\sum_{n=1}^{\infty} \sup \left\{\left\|S_{n} x-S_{n-1} x\right\|: x \in C\right\}<$ $\infty$. Then for each $y \in C,\left\{S_{n} y\right\}$ converges strongly to some point of $C$. Moreover, let $S$ be a mapping of $C$ into itself defined by $S y=\lim _{n \rightarrow \infty} S_{n} y$ for all $y \in C$. Then $\lim _{n \rightarrow \infty} \sup \left\{\left\|S x-S_{n} x\right\|:\right.$ $x \in C\}=0$.

\section{Mann-Type Extragradient Algorithms in Uniformly Convex and 2-Uniformly Smooth Banach Spaces}

In this section, we introduce Mann-type extragradient algorithms in uniformly convex and 2-uniformly smooth Banach spaces and show weak and strong convergence theorems. We will use some useful lemmas in the sequel.

Lemma 12 (see [2, Lemma 2.8]). Let $C$ be a nonempty closed convex subset of a real 2-uniformly smooth Banach space $X$. Let the mapping $B_{i}: C \rightarrow X$ be $\alpha_{i}$-inverse-strongly accretive. Then, we have

$$
\begin{array}{r}
\left\|\left(I-\mu_{i} B_{i}\right) x-\left(I-\mu_{i} B_{i}\right) y\right\|^{2} \\
\leq\|x-y\|^{2}+2 \mu_{i}\left(\mu_{i} \kappa^{2}-\alpha_{i}\right)\left\|B_{i} x-B_{i} y\right\|^{2}, \\
\forall x, y \in C,
\end{array}
$$

for $i=1,2$, where $\mu_{i}>0$. In particular, if $0<\mu_{i} \leq \alpha_{i} / \kappa^{2}$, then $I-\mu_{i} B_{i}$ is nonexpansive for $i=1,2$.

Lemma 13 (see [2, Lemma 2.9]). Let $C$ be a nonempty closed convex subset of a real 2-uniformly smooth Banach space X. 
Let $\Pi_{C}$ be a sunny nonexpansive retraction from $X$ onto $C$. Let the mapping $B_{i}: C \rightarrow X$ be $\alpha_{i}$-inverse-strongly accretive for $i=1,2$. Let $G: C \rightarrow C$ be the mapping defined by

$$
\begin{array}{r}
G x=\Pi_{C}\left[\Pi_{C}\left(x-\mu_{2} B_{2} x\right)-\mu_{1} B_{1} \Pi_{C}\left(x-\mu_{2} B_{2} x\right)\right], \\
\forall x \in C .
\end{array}
$$

If $0<\mu_{i} \leq \alpha_{i} / \kappa^{2}$ for $i=1,2$, then $G: C \rightarrow C$ is nonexpansive.

Theorem 14. Let $X$ be a uniformly convex and 2-uniformly smooth Banach space satisfying Opial's condition and let $C$ be a nonempty closed convex subset of $X$ such that $C \pm C \subset C$. Let $\Pi_{C}$ be a sunny nonexpansive retraction from $X$ onto $C$. Let $T$ and $F: X \rightarrow C B(X)$ and let $A: C \rightarrow 2^{C}$ be three multivalued mappings, let $g: X \rightarrow C$ be a single-valued mapping, and let $N(\cdot, \cdot): X \times X \rightarrow C$ be a single-valued continuous mapping satisfying conditions (C1)-(C5) in Theorem 2 and

(C6) $N(T x, F x)+A(g(x)): X \rightarrow 2^{C} \backslash\{\emptyset\}$ is $\zeta$-inverse strongly accretive with $\zeta \geq \kappa^{2}$.

Let $B_{i}: C \rightarrow X$ be $\alpha_{i}$-inverse strongly accretive for $i=1,2$. Let $\left\{S_{i}\right\}_{i=0}^{\infty}$ be a countable family of nonexpansive mappings of C into itself such that $\Delta:=\bigcap_{i=0}^{\infty} \operatorname{Fix}\left(S_{i}\right) \cap \Omega \cap \Gamma \neq \emptyset$, where $\Omega$ is the fixed point set of the mapping $G=\Pi_{C}\left(I-\mu_{1} B_{1}\right) \Pi_{C}\left(I-\mu_{2} B_{2}\right)$ with $0<\mu_{i}<\alpha_{i} / \kappa^{2}$ for $i=1,2$. Assume that $\left\{\alpha_{n}\right\},\left\{\beta_{n}\right\}$, and $\left\{\sigma_{n}\right\}$ are sequences in $[0,1]$ such that

(i) $0<\liminf _{n \rightarrow \infty} \alpha_{n} \leq \limsup _{n \rightarrow \infty} \alpha_{n}<1$;

(ii) $0<\liminf _{n \rightarrow \infty} \beta_{n} \leq \lim \sup _{n \rightarrow \infty} \beta_{n}<1$;

(iii) $0<\liminf _{n \rightarrow \infty} \sigma_{n} \leq \limsup _{n \rightarrow \infty} \sigma_{n}<1$.

For arbitrary $x_{0} \in C$ define the sequence $\left\{x_{n}\right\}$ iteratively by

$$
\begin{gathered}
y_{n}=\beta_{n} S_{n} x_{n}+\left(1-\beta_{n}\right) \Pi_{C}\left(I-\mu_{1} B_{1}\right) \\
\times \Pi_{C}\left(I-\mu_{2} B_{2}\right) x_{n}, \\
x_{n+1}=\alpha_{n}\left[x_{n}-\sigma_{n}\left(N\left(w_{n}, k_{n}\right)+u_{n}\right)\right]+\left(1-\alpha_{n}\right) y_{n}, \\
u_{n} \in A\left(g\left(x_{n}\right)\right), \quad \forall n \geq 0,
\end{gathered}
$$

where $\left\{u_{n}\right\}$ is defined by

$$
\begin{array}{r}
\left\|u_{n}-u_{n+1}\right\| \leq(1+\varepsilon) H\left(A\left(g\left(x_{n+1}\right)\right), A\left(g\left(x_{n}\right)\right)\right), \\
\forall n \geq 0,
\end{array}
$$

for any $w_{n} \in T x_{n}, k_{n} \in F x_{n}$ and some $\varepsilon>0$. Assume that $\sum_{n=0}^{\infty} \sup _{x \in D}\left\|S_{n+1} x-S_{n} x\right\|<\infty$ for any bounded subset $D$ of $C$ and let $S$ be a mapping of $C$ into itself defined by $S x=\lim _{n \rightarrow \infty} S_{n} x$ for all $x \in C$ and suppose that $\operatorname{Fix}(S)=$ $\bigcap_{i=0}^{\infty} \operatorname{Fix}\left(S_{i}\right)$. Then $\left\{x_{n}\right\}$ converges weakly to some $\bar{x} \in \Delta$, and for any $w \in T \bar{x}, k \in F \bar{x},(\bar{x}, w, k)$ is a solution of the MVVI (15).

Proof. First of all, let us show that for any $v \in C, \lambda>0$, there exists a point $\tilde{x} \in C$ such that $(\tilde{x}, w, k)$ is a solution of the MVVI (14), for any $w \in T \tilde{x}$ and $k \in F \tilde{x}$. Indeed, following the argument idea in the proof of Chidume et al. [4, Theorem 3.1], we put $V x:=N(T x, F x)$ for all $x \in X$. Then by Proposition $4, V$ is $\phi$-strongly accretive. Since $T$ and $F$ are $H$-uniformly continuous and $N(\cdot, \cdot)$ is continuous, $V x$ is continuous and hence lower semicontinuous. Thus, by Proposition $5 V x$ is single-valued. Moreover, since $V$ is $\phi$ strongly accretive and by assumption $A \circ g: X \rightarrow 2^{C}$ is $m$-accretive, we have that $V+\lambda A \circ g$ is an $m$-accretive and $\phi$-strongly accretive mapping, and hence by Cioranescu [18, page 184] for any $x \in X$ we have $(V+\lambda A \circ g)(x)$ is closed and bounded. Therefore, by Morales [19], $V+\lambda A \circ g$ is surjective. Hence, for any $v \in X$ and $\lambda>0$ there exists $\tilde{x} \in D(A)=C$ such that $v \in V \tilde{x}+\lambda A(g(\tilde{x}))=N(w, k)+$ $\lambda A(g(\tilde{x}))$, where $w \in T \tilde{x}$ and $k \in F \tilde{x}$. In addition, in terms of Proposition 5 we know that $V+\lambda A \circ g$ is a single-valued mapping. Assume that $N(T x, F x)+\lambda A(g(x)): X \rightarrow C$ is $\zeta$ inverse strongly accretive with $\zeta \geq \kappa^{2}$. Then by Lemma 12 , we conclude that the mapping $x \mapsto x-(N(T x, F x)+\lambda A(g(x)))$ is nonexpansive. Meantime, by Lemma 13 we know that $G$ : $C \rightarrow C$ is also nonexpansive.

Without loss of generality we may assume that $v=0$ and $\lambda=1$. Let $p \in \Delta$ and let $r>0$ be sufficiently large such that $x_{0} \in \bar{B}_{r}(p)=: B$. Then $p \in D(A)=C$ such that $0 \in N(w, k)+$ $A \circ g(p)$ for any $w \in T p$ and $k \in F p$. Let $M:=\sup \{\|u\|$ : $u \in N(w, k)+A(g(x)), x \in B, w \in T x, k \in F x\}$. Then as $A \circ g, T$ and $F$ are $H$-uniformly continuous on $X$, for $\varepsilon_{1}:=$ $\phi(r) / 8(1+\varepsilon)$, and $\varepsilon_{2}:=\phi(r) / 8 \mu(1+\varepsilon), \varepsilon_{3}:=\phi(r) / 8 \xi(1+\varepsilon)$, there exist $\delta_{1}, \delta_{2}, \delta_{3}>0$ such that for any $x, y \in X,\|x-y\|<$ $\delta_{1},\|x-y\|<\delta_{2}$ and $\|x-y\|<\delta_{3}$ imply $H(A \circ g(x), A \circ g(y))<$ $\varepsilon_{1}, H(T x, T y)<\varepsilon_{2}$ and $H(F x, F y)<\varepsilon_{3}$, respectively.

Let us show that $x_{n} \in B$ for all $n \geq 0$. We show this by induction. First, $x_{0} \in B$ by construction. Assume that $x_{n} \in B$. We show that $x_{n+1} \in B$. If possible we assume that $x_{n+1} \notin B$, then $\left\|x_{n+1}-p\right\|>r$. Further from (32) it follows that

$$
\begin{aligned}
\left\|y_{n}-p\right\|= & \| \beta_{n} S_{n} x_{n}+\left(1-\beta_{n}\right) \Pi_{C}\left(I-\mu_{1} B_{1}\right) \Pi_{C} \\
& \times\left(I-\mu_{2} B_{2}\right) x_{n}-p \| \\
\leq & \beta_{n}\left\|S_{n} x_{n}-p\right\|+\left(1-\beta_{n}\right)\left\|G x_{n}-p\right\| \\
\leq & \beta_{n}\left\|x_{n}-p\right\|+\left(1-\beta_{n}\right)\left\|x_{n}-p\right\| \\
= & \left\|x_{n}-p\right\|,
\end{aligned}
$$

and hence

$$
\begin{aligned}
& \left\|x_{n+1}-p\right\|^{2} \\
& =\left\langle\alpha_{n}\left[x_{n}-p-\sigma_{n}\left(N\left(w_{n}, k_{n}\right)+u_{n}\right)\right]\right. \\
& \left.+\left(1-\alpha_{n}\right)\left(y_{n}-p\right), J\left(x_{n+1}-p\right)\right\rangle \\
& =\left\langle\alpha_{n}\left(x_{n}-p\right)+\left(1-\alpha_{n}\right)\right. \\
& \left.\quad \times\left(y_{n}-p\right), J\left(x_{n+1}-p\right)\right\rangle \\
& \quad-\alpha_{n} \sigma_{n}\left\langle N\left(w_{n}, k_{n}\right)+u_{n}, J\left(x_{n+1}-p\right)\right\rangle
\end{aligned}
$$




$$
\begin{aligned}
\leq & \left\|\alpha_{n}\left(x_{n}-p\right)+\left(1-\alpha_{n}\right)\left(y_{n}-p\right)\right\| \\
& \times\left\|x_{n+1}-p\right\| \\
& -\alpha_{n} \sigma_{n}\left\langle N\left(w_{n}, k_{n}\right)+u_{n}, J\left(x_{n+1}-p\right)\right\rangle \\
\leq & \left(\alpha_{n}\left\|x_{n}-p\right\|+\left(1-\alpha_{n}\right)\left\|x_{n}-p\right\|\right) \\
& \times\left\|x_{n+1}-p\right\| \\
& -\alpha_{n} \sigma_{n}\left\langle N\left(w_{n}, k_{n}\right)+u_{n}, J\left(x_{n+1}-p\right)\right\rangle \\
= & \left\|x_{n}-p\right\|\left\|x_{n+1}-p\right\| \\
& -\alpha_{n} \sigma_{n}\left\langle N\left(w_{n}, k_{n}\right)+u_{n}, J\left(x_{n+1}-p\right)\right\rangle \\
\leq & \frac{1}{2}\left(\left\|x_{n}-p\right\|^{2}+\left\|x_{n+1}-p\right\|^{2}\right) \\
& -\alpha_{n} \sigma_{n}\left\langle N\left(w_{n}, k_{n}\right)+u_{n}, J\left(x_{n+1}-p\right)\right\rangle,
\end{aligned}
$$

which immediately yields

$$
\begin{aligned}
& \left\|x_{n+1}-p\right\|^{2} \\
& \leq\left\|x_{n}-p\right\|^{2}-2 \alpha_{n} \sigma_{n}\left\langle N\left(w_{n}, k_{n}\right)+u_{n}, J\left(x_{n+1}-p\right)\right\rangle \\
& =\left\|x_{n}-p\right\|^{2} \\
& -2 \alpha_{n} \sigma_{n}\left\langle N\left(w_{n+1}, k_{n+1}\right)+u_{n+1}, J\left(x_{n+1}-p\right)\right\rangle \\
& -2 \alpha_{n} \sigma_{n}\left\langle N\left(w_{n}, k_{n}\right)\right. \\
& +u_{n}-\left(N\left(w_{n+1}, k_{n+1}\right)+u_{n+1}\right) \\
& \left.J\left(x_{n+1}-p\right)\right\rangle .
\end{aligned}
$$

Since $N(\cdot, \cdot)$ is $\phi$-strongly accretive with respect to $T$ and $A(g(\cdot))$ is accretive, we deduce from (36) that

$$
\begin{aligned}
& \left\|x_{n+1}-p\right\|^{2} \\
& \leq\left\|x_{n}-p\right\|^{2}-2 \alpha_{n} \sigma_{n} \phi\left(\left\|x_{n+1}-p\right\|\right)\left\|x_{n+1}-p\right\| \\
& +2 \alpha_{n} \sigma_{n}\left[\left\|N\left(w_{n+1}, k_{n+1}\right)-N\left(w_{n}, k_{n}\right)\right\|\right. \\
& \left.+\left\|u_{n+1}-u_{n}\right\|\right]\left\|x_{n+1}-p\right\| \\
& \leq\left\|x_{n}-p\right\|^{2}-2 \alpha_{n} \sigma_{n} \phi\left(\left\|x_{n+1}-p\right\|\right)\left\|x_{n+1}-p\right\| \\
& +2 \alpha_{n} \sigma_{n}\left[\left\|N\left(w_{n+1}, k_{n+1}\right)-N\left(w_{n+1}, k_{n}\right)\right\|\right. \\
& +\left\|N\left(w_{n+1}, k_{n}\right)-N\left(w_{n}, k_{n}\right)\right\| \\
& \left.+\left\|u_{n+1}-u_{n}\right\|\right]\left\|x_{n+1}-p\right\| .
\end{aligned}
$$

Again from (32) we have that

$$
\begin{aligned}
&\left\|x_{n+1}-p\right\| \\
& \leq \alpha_{n}\left\|x_{n}-p-\sigma_{n}\left(N\left(w_{n}, k_{n}\right)+u_{n}\right)\right\| \\
&+\left(1-\alpha_{n}\right)\left\|y_{n}-p\right\| \\
& \leq \alpha_{n}\left[\left\|x_{n}-p\right\|+\sigma_{n}\left\|N\left(w_{n}, k_{n}\right)+u_{n}\right\|\right] \\
&+\left(1-\alpha_{n}\right)\left\|x_{n}-p\right\| \\
& \leq \alpha_{n}\left[r+\sigma_{n} M\right]+\left(1-\alpha_{n}\right) r \\
& \leq 2 r .
\end{aligned}
$$

Also, from Proposition 5, $V x=N(T x, F x)$ is a single-valued mapping; that is, for any $k, k^{\prime} \in F x$ and $w, w^{\prime} \in T x$ we have $N(w, k)=N\left(w, k^{\prime}\right)$ and $N(w, k)=N\left(w^{\prime}, k\right)$. On the other hand, it follows from Nadler [20] that, for $k_{n+1} \in F x_{n+1}$ and $w_{n+1} \in T x_{n+1}$, there exist $k_{n}^{\prime} \in F x_{n}$ and $w_{n}^{\prime} \in T x_{n}$ such that

$$
\begin{gathered}
\left\|k_{n+1}-k_{n}^{\prime}\right\| \leq(1+\varepsilon) H\left(F x_{n+1}, F x_{n}\right), \\
\left\|w_{n+1}-w_{n}^{\prime}\right\| \leq(1+\varepsilon) H\left(T x_{n+1}, T x_{n}\right),
\end{gathered}
$$

respectively. Therefore, from (37) and (33), we have that

$$
\begin{aligned}
& \left\|x_{n+1}-p\right\|^{2} \\
& \leq\left\|x_{n}-p\right\|^{2}-2 \alpha_{n} \sigma_{n} \phi(r) r \\
& +2 \alpha_{n} \sigma_{n}\left[\left\|N\left(w_{n+1}, k_{n+1}\right)-N\left(w_{n+1}, k_{n}^{\prime}\right)\right\|\right. \\
& +\left\|N\left(w_{n+1}, k_{n}\right)-N\left(w_{n}^{\prime}, k_{n}\right)\right\| \\
& \left.+\left\|u_{n+1}-u_{n}\right\|\right] 2 r \\
& \leq\left\|x_{n}-p\right\|^{2}-2 \alpha_{n} \sigma_{n} \phi(r) r \\
& +2 \alpha_{n} \sigma_{n}\left[\xi(1+\varepsilon) H\left(F x_{n+1}, F x_{n}\right)\right. \\
& +\mu(1+\varepsilon) H\left(T x_{n+1}, T x_{n}\right) \\
& \left.+(1+\varepsilon) H\left(A\left(g\left(x_{n+1}\right)\right), A\left(g\left(x_{n}\right)\right)\right)\right] 2 r \\
& \leq\left\|x_{n}-p\right\|^{2}-2 \alpha_{n} \sigma_{n} \phi(r) r \\
& +2 \alpha_{n} \sigma_{n}\left[\frac{\phi(r)}{8}+\frac{\phi(r)}{8}+\frac{\phi(r)}{8}\right] 2 r \\
& =\left\|x_{n}-p\right\|^{2}-2 \alpha_{n} \sigma_{n} \phi(r) r \\
& +\alpha_{n} \frac{3}{2} \phi(r) r \leq\left\|x_{n}-p\right\|^{2} .
\end{aligned}
$$

So, we get $\left\|x_{n+1}-p\right\| \leq r$, a contradiction. Therefore, $\left\{x_{n}\right\}$ is bounded.

Let us show that $\lim _{n \rightarrow \infty}\left\|x_{n}-y_{n}\right\|=0$ and $\lim _{n \rightarrow \infty} \| x_{n}-$ $x_{n+1} \|=0$. 
Indeed, utilizing Lemma 10 and the nonexpansivity of the mapping $x \mapsto x-(N(T x, F x)+A(g(x)))$, we obtain from (32) that for all $n \geq 0$

$$
\begin{aligned}
& \left\|x_{n+1}-p\right\|^{2} \\
& =\| \alpha_{n}\left[x_{n}-p-\sigma_{n}\left(N\left(w_{n}, k_{n}\right)+u_{n}\right)\right] \\
& +\left(1-\alpha_{n}\right)\left(y_{n}-p\right) \|^{2} \\
& \leq \alpha_{n}\left\|x_{n}-p-\sigma_{n}\left(N\left(w_{n}, k_{n}\right)+u_{n}\right)\right\|^{2} \\
& +\left(1-\alpha_{n}\right)\left\|y_{n}-p\right\|^{2}-\alpha_{n}\left(1-\alpha_{n}\right) \varphi \\
& \times\left(\left\|x_{n}-y_{n}-\sigma_{n}\left(N\left(w_{n}, k_{n}\right)+u_{n}\right)\right\|\right) \\
& =\alpha_{n} \|\left(1-\sigma_{n}\right)\left(x_{n}-p\right) \\
& +\sigma_{n}\left[x_{n}-\left(N\left(w_{n}, k_{n}\right)+u_{n}\right)-p\right] \|^{2} \\
& +\left(1-\alpha_{n}\right)\left\|y_{n}-p\right\|^{2}-\alpha_{n}\left(1-\alpha_{n}\right) \varphi \\
& \times\left(\left\|x_{n}-y_{n}-\sigma_{n}\left(N\left(w_{n}, k_{n}\right)+u_{n}\right)\right\|\right) \\
& \leq \alpha_{n}\left[\left(1-\sigma_{n}\right)\left\|x_{n}-p\right\|^{2}\right. \\
& +\sigma_{n}\left\|x_{n}-\left(N\left(w_{n}, k_{n}\right)+u_{n}\right)-p\right\|^{2} \\
& \left.-\sigma_{n}\left(1-\sigma_{n}\right) \varphi_{1}\left(\left\|N\left(w_{n}, k_{n}\right)+u_{n}\right\|\right)\right] \\
& +\left(1-\alpha_{n}\right)\left\|x_{n}-p\right\|^{2}-\alpha_{n}\left(1-\alpha_{n}\right) \varphi \\
& \times\left(\left\|x_{n}-y_{n}-\sigma_{n}\left(N\left(w_{n}, k_{n}\right)+u_{n}\right)\right\|\right) \\
& \leq \alpha_{n}\left[\left(1-\sigma_{n}\right)\left\|x_{n}-p\right\|^{2}+\sigma_{n}\left\|x_{n}-p\right\|^{2}\right. \\
& \left.-\sigma_{n}\left(1-\sigma_{n}\right) \varphi_{1}\left(\left\|N\left(w_{n}, k_{n}\right)+u_{n}\right\|\right)\right] \\
& +\left(1-\alpha_{n}\right)\left\|x_{n}-p\right\|^{2}-\alpha_{n}\left(1-\alpha_{n}\right) \varphi \\
& \times\left(\left\|x_{n}-y_{n}-\sigma_{n}\left(N\left(w_{n}, k_{n}\right)+u_{n}\right)\right\|\right) \\
& =\alpha_{n}\left\|x_{n}-p\right\|^{2}-\alpha_{n} \sigma_{n}\left(1-\sigma_{n}\right) \varphi_{1} \\
& \times\left(\left\|N\left(w_{n}, k_{n}\right)+u_{n}\right\|\right) \\
& +\left(1-\alpha_{n}\right)\left\|x_{n}-p\right\|^{2}-\alpha_{n}\left(1-\alpha_{n}\right) \varphi \\
& \times\left(\left\|x_{n}-y_{n}-\sigma_{n}\left(N\left(w_{n}, k_{n}\right)+u_{n}\right)\right\|\right) \\
& =\left\|x_{n}-p\right\|^{2} \\
& -\alpha_{n} \sigma_{n}\left(1-\sigma_{n}\right) \varphi_{1}\left(\left\|N\left(w_{n}, k_{n}\right)+u_{n}\right\|\right) \\
& -\alpha_{n}\left(1-\alpha_{n}\right) \varphi \\
& \times\left(\left\|x_{n}-y_{n}-\sigma_{n}\left(N\left(w_{n}, k_{n}\right)+u_{n}\right)\right\|\right) \\
& \leq\left\|x_{n}-p\right\|^{2} \text {. }
\end{aligned}
$$

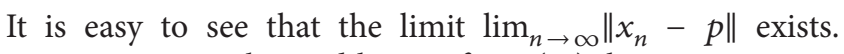
Meantime, it can be readily seen from (41) that

$$
\begin{aligned}
\alpha_{n} \sigma_{n} & \left(1-\sigma_{n}\right) \varphi_{1}\left(\left\|N\left(w_{n}, k_{n}\right)+u_{n}\right\|\right) \\
& +\alpha_{n}\left(1-\alpha_{n}\right) \varphi \\
& \times\left(\left\|x_{n}-y_{n}-\sigma_{n}\left(N\left(w_{n}, k_{n}\right)+u_{n}\right)\right\|\right) \\
\leq & \left\|x_{n}-p\right\|^{2}-\left\|x_{n+1}-p\right\|^{2},
\end{aligned}
$$

which together with conditions (i) and (iii) and the existence of $\lim _{n \rightarrow \infty}\left\|x_{n}-p\right\|$, implies that

$$
\begin{gathered}
\lim _{n \rightarrow \infty} \varphi_{1}\left(\left\|N\left(w_{n}, k_{n}\right)+u_{n}\right\|\right)=0, \\
\lim _{n \rightarrow \infty} \varphi\left(\left\|x_{n}-y_{n}-\sigma_{n}\left(N\left(w_{n}, k_{n}\right)+u_{n}\right)\right\|\right)=0 .
\end{gathered}
$$

Utilizing the properties of $\varphi$ and $\varphi_{1}$, we get

$$
\begin{gathered}
\lim _{n \rightarrow \infty}\left\|N\left(w_{n}, k_{n}\right)+u_{n}\right\|=0, \\
\lim _{n \rightarrow \infty}\left\|x_{n}-y_{n}-\sigma_{n}\left(N\left(w_{n}, k_{n}\right)+u_{n}\right)\right\|=0 .
\end{gathered}
$$

Note that

$$
\begin{aligned}
\left\|x_{n}-y_{n}\right\| \leq & \left\|x_{n}-y_{n}-\sigma_{n}\left(N\left(w_{n}, k_{n}\right)+u_{n}\right)\right\| \\
& +\sigma_{n}\left\|N\left(w_{n}, k_{n}\right)+u_{n}\right\| .
\end{aligned}
$$

So, we have

$$
\lim _{n \rightarrow \infty}\left\|x_{n}-y_{n}\right\|=0
$$

Also, observe that

$$
\begin{aligned}
\left\|x_{n+1}-x_{n}\right\| \leq & \alpha_{n} \sigma_{n}\left\|N\left(w_{n}, k_{n}\right)+u_{n}\right\| \\
& +\left(1-\alpha_{n}\right)\left\|x_{n}-y_{n}\right\| \\
\leq & \left\|N\left(w_{n}, k_{n}\right)+u_{n}\right\|+\left\|x_{n}-y_{n}\right\| .
\end{aligned}
$$

Thus, from (44) and (46) it follows that

$$
\lim _{n \rightarrow \infty}\left\|x_{n+1}-x_{n}\right\|=0
$$

Let us show that $\lim _{n \rightarrow \infty}\left\|x_{n}-G x_{n}\right\|=\lim _{n \rightarrow \infty} \| x_{n}-$ $S x_{n} \|=0$. 
Indeed, for simplicity, put $q=\Pi_{C}\left(p-\mu_{2} B_{2} p\right), u_{n}=$ $\Pi_{C}\left(x_{n}-\mu_{2} B_{2} x_{n}\right)$ and $v_{n}=\Pi_{C}\left(u_{n}-\mu_{1} B_{1} u_{n}\right)$. Then $v_{n}=G x_{n}$ for all $n \geq 0$. From Lemma 12 we have

$$
\begin{aligned}
&\left\|u_{n}-q\right\|^{2} \\
&=\left\|\Pi_{C}\left(x_{n}-\mu_{2} B_{2} x_{n}\right)-\Pi_{C}\left(p-\mu_{2} B_{2} p\right)\right\|^{2} \\
& \leq\left\|x_{n}-p-\mu_{2}\left(B_{2} x_{n}-B_{2} p\right)\right\|^{2} \\
& \leq\left\|x_{n}-p\right\|^{2}-2 \mu_{2}\left(\alpha_{2}-\kappa^{2} \mu_{2}\right) \\
& \times\left\|B_{2} x_{n}-B_{2} p\right\|^{2}, \\
& \| v_{n}- p \|^{2} \\
&=\left\|\Pi_{C}\left(u_{n}-\mu_{1} B_{1} u_{n}\right)-\Pi_{C}\left(q-\mu_{1} B_{1} q\right)\right\|^{2} \\
& \leq\left\|u_{n}-q-\mu_{1}\left(B_{1} u_{n}-B_{1} q\right)\right\|^{2} \\
& \leq\left\|u_{n}-q\right\|^{2}-2 \mu_{1}\left(\alpha_{1}-\kappa^{2} \mu_{1}\right) \\
& \times\left\|B_{1} u_{n}-B_{1} q\right\|^{2} .
\end{aligned}
$$

Substituting (49) for (50), we obtain

$$
\begin{aligned}
\| v_{n}- & p \|^{2} \\
\leq & \left\|x_{n}-p\right\|^{2} \\
& -2 \mu_{2}\left(\alpha_{2}-\kappa^{2} \mu_{2}\right)\left\|B_{2} x_{n}-B_{2} p\right\|^{2} \\
& -2 \mu_{1}\left(\alpha_{1}-\kappa^{2} \mu_{1}\right)\left\|B_{1} u_{n}-B_{1} q\right\|^{2} .
\end{aligned}
$$

Utilizing [21, Proposition 1] and Lemma 10, from (32) and (51) we have

$$
\begin{aligned}
& \left\|y_{n}-p\right\|^{2} \\
& \leq \beta_{n}\left\|S_{n} x_{n}-p\right\|^{2}+\left(1-\beta_{n}\right)\left\|G x_{n}-p\right\|^{2} \\
& \quad-\beta_{n}\left(1-\beta_{n}\right) \varphi_{2}\left(\left\|S_{n} x_{n}-G x_{n}\right\|\right) \\
& \leq \beta_{n}\left\|x_{n}-p\right\|^{2}+\left(1-\beta_{n}\right)\left\|v_{n}-p\right\|^{2} \\
& \quad-\beta_{n}\left(1-\beta_{n}\right) \varphi_{2}\left(\left\|S_{n} x_{n}-G x_{n}\right\|\right) \\
& \leq \beta_{n}\left\|x_{n}-p\right\|^{2} \\
& \quad-\beta_{n}\left(1-\beta_{n}\right) \varphi_{2}\left(\left\|S_{n} x_{n}-G x_{n}\right\|\right) \\
& \quad+\left(1-\beta_{n}\right)\left[\left\|x_{n}-p\right\|^{2}\right. \\
& \quad-2 \mu_{2}\left(\alpha_{2}-\kappa^{2} \mu_{2}\right)\left\|B_{2} x_{n}-B_{2} p\right\|^{2} \\
& \left.\quad-2 \mu_{1}\left(\alpha_{1}-\kappa^{2} \mu_{1}\right)\left\|B_{1} u_{n}-B_{1} q\right\|^{2}\right]
\end{aligned}
$$

$$
\begin{aligned}
& =\left\|x_{n}-p\right\|^{2} \\
& -\beta_{n}\left(1-\beta_{n}\right) \varphi_{2}\left(\left\|S_{n} x_{n}-G x_{n}\right\|\right) \\
& -2\left(1-\beta_{n}\right)\left[\mu_{2}\left(\alpha_{2}-\kappa^{2} \mu_{2}\right)\left\|B_{2} x_{n}-B_{2} p\right\|^{2}\right. \\
& \left.\quad+2 \mu_{1}\left(\alpha_{1}-\kappa^{2} \mu_{1}\right)\left\|B_{1} u_{n}-B_{1} q\right\|^{2}\right],
\end{aligned}
$$

which hence implies that

$$
\begin{array}{r}
\beta_{n}\left(1-\beta_{n}\right) \varphi_{2}\left(\left\|S_{n} x_{n}-G x_{n}\right\|\right) \\
+2\left(1-\beta_{n}\right)\left[\mu_{2}\left(\alpha_{2}-\kappa^{2} \mu_{2}\right)\right. \\
\times\left\|B_{2} x_{n}-B_{2} p\right\|^{2} \\
+\mu_{1}\left(\alpha_{1}-\kappa^{2} \mu_{1}\right) \\
\left.\times\left\|B_{1} u_{n}-B_{1} q\right\|^{2}\right] \\
\leq\left\|x_{n}-p\right\|^{2}-\left\|y_{n}-p\right\|^{2} \\
\leq\left(\left\|x_{n}-p\right\|+\left\|y_{n}-p\right\|\right)\left\|x_{n}-y_{n}\right\| .
\end{array}
$$

Since $0<\mu_{i}<\alpha_{i} / \kappa^{2}$ for $i=1,2$ and $\left\{x_{n}\right\},\left\{y_{n}\right\}$ are bounded, we obtain from (46), (53), condition (ii) and the properties of $\varphi_{2}$ that

$$
\begin{gathered}
\lim _{n \rightarrow \infty}\left\|B_{2} x_{n}-B_{2} p\right\|=\lim _{n \rightarrow \infty}\left\|B_{1} u_{n}-B_{1} q\right\|=0, \\
\lim _{n \rightarrow \infty}\left\|S_{n} x_{n}-G x_{n}\right\|=0 .
\end{gathered}
$$

Utilizing Proposition 3 and Lemma 8, we have

$$
\begin{aligned}
&\left\|u_{n}-q\right\|^{2} \\
&=\left\|\Pi_{C}\left(x_{n}-\mu_{2} B_{2} x_{n}\right)-\Pi_{C}\left(p-\mu_{2} B_{2} p\right)\right\|^{2} \\
& \leq\left\langle x_{n}-\mu_{2} B_{2} x_{n}-\left(p-\mu_{2} B_{2} p\right), J\left(u_{n}-q\right)\right\rangle \\
&=\left\langle x_{n}-p, J\left(u_{n}-q\right)\right\rangle \\
&+\mu_{2}\left\langle B_{2} p-B_{2} x_{n}, J\left(u_{n}-q\right)\right\rangle \\
& \leq \frac{1}{2}\left[\left\|x_{n}-p\right\|^{2}+\left\|u_{n}-q\right\|^{2}\right. \\
&\left.\quad-\psi_{1}\left(\left\|x_{n}-u_{n}-(p-q)\right\|\right)\right] \\
&+\mu_{2}\left\|B_{2} p-B_{2} x_{n}\right\|\left\|u_{n}-q\right\|,
\end{aligned}
$$

which implies that

$$
\begin{aligned}
\left\|u_{n}-q\right\|^{2} \leq & \left\|x_{n}-p\right\|^{2} \\
& -\psi_{1}\left(\left\|x_{n}-u_{n}-(p-q)\right\|\right) \\
& +2 \mu_{2}\left\|B_{2} p-B_{2} x_{n}\right\|\left\|u_{n}-q\right\| .
\end{aligned}
$$


In the same way, we derive

$$
\begin{aligned}
& \| v_{n}- p \|^{2} \\
&=\left\|\Pi_{C}\left(u_{n}-\mu_{1} B_{1} u_{n}\right)-\Pi_{C}\left(q-\mu_{1} B_{1} q\right)\right\|^{2} \\
& \leq\left\langle u_{n}-\mu_{1} B_{1} u_{n}-\left(q-\mu_{1} B_{1} q\right), J\left(v_{n}-p\right)\right\rangle \\
&=\left\langle u_{n}-q, J\left(v_{n}-p\right)\right\rangle \\
&+\mu_{1}\left\langle B_{1} q-B_{1} u_{n}, J\left(v_{n}-p\right)\right\rangle \\
& \leq \frac{1}{2}\left[\left\|u_{n}-q\right\|^{2}+\left\|v_{n}-p\right\|^{2}\right. \\
&\left.\quad-\psi_{2}\left(\left\|u_{n}-v_{n}+(p-q)\right\|\right)\right] \\
&+\mu_{1}\left\|B_{1} q-B_{1} u_{n}\right\|\left\|v_{n}-p\right\|,
\end{aligned}
$$

which implies that

$$
\begin{aligned}
\left\|v_{n}-p\right\|^{2} \leq & \left\|u_{n}-q\right\|^{2} \\
& -\psi_{2}\left(\left\|u_{n}-v_{n}+(p-q)\right\|\right) \\
& +2 \mu_{1}\left\|B_{1} q-B_{1} u_{n}\right\|\left\|v_{n}-p\right\| .
\end{aligned}
$$

Substituting (56) for (58), we get

$$
\begin{aligned}
\left\|v_{n}-p\right\|^{2} \leq & \left\|x_{n}-p\right\|^{2} \\
& -\psi_{1}\left(\left\|x_{n}-u_{n}-(p-q)\right\|\right) \\
& -\psi_{2}\left(\left\|u_{n}-v_{n}+(p-q)\right\|\right) \\
& +2 \mu_{2}\left\|B_{2} p-B_{2} x_{n}\right\|\left\|u_{n}-q\right\| \\
& +2 \mu_{1}\left\|B_{1} q-B_{1} u_{n}\right\|\left\|v_{n}-p\right\| .
\end{aligned}
$$

By Lemma 7, we have from (52) and (59)

$$
\begin{aligned}
& \left\|y_{n}-p\right\|^{2} \\
& \leq \beta_{n}\left\|S_{n} x_{n}-p\right\|^{2}+\left(1-\beta_{n}\right)\left\|v_{n}-p\right\|^{2} \\
& \leq \beta_{n}\left\|x_{n}-p\right\|^{2}+\left(1-\beta_{n}\right) \\
& \quad \times\left[\left\|x_{n}-p\right\|^{2}\right. \\
& \quad-\psi_{1}\left(\left\|x_{n}-u_{n}-(p-q)\right\|\right) \\
& \quad-\psi_{2}\left(\left\|u_{n}-v_{n}+(p-q)\right\|\right) \\
& \quad+2 \mu_{2}\left\|B_{2} p-B_{2} x_{n}\right\|\left\|u_{n}-q\right\| \\
& \left.\quad+2 \mu_{1}\left\|B_{1} q-B_{1} u_{n}\right\|\left\|v_{n}-p\right\|\right]
\end{aligned}
$$

$$
\begin{aligned}
\leq & \left\|x_{n}-p\right\|^{2}-\left(1-\beta_{n}\right) \\
& \times\left[\psi_{1}\left(\left\|x_{n}-u_{n}-(p-q)\right\|\right)\right. \\
& \left.+\psi_{2}\left(\left\|u_{n}-v_{n}+(p-q)\right\|\right)\right] \\
& +2 \mu_{2}\left\|B_{2} p-B_{2} x_{n}\right\|\left\|u_{n}-q\right\| \\
& +2 \mu_{1}\left\|B_{1} q-B_{1} u_{n}\right\|\left\|v_{n}-p\right\|,
\end{aligned}
$$

which hence leads to

$$
\begin{gathered}
\left(1-\beta_{n}\right)\left[\psi_{1}\left(\left\|x_{n}-u_{n}-(p-q)\right\|\right)\right. \\
\left.+\psi_{2}\left(\left\|u_{n}-v_{n}+(p-q)\right\|\right)\right] \\
\leq\left\|x_{n}-p\right\|^{2}-\left\|y_{n}-p\right\|^{2} \\
+2 \mu_{2}\left\|B_{2} p-B_{2} x_{n}\right\|\left\|u_{n}-q\right\| \\
+2 \mu_{1}\left\|B_{1} q-B_{1} u_{n}\right\|\left\|v_{n}-p\right\| \\
\leq \\
\left(\left\|x_{n}-p\right\|+\left\|y_{n}-p\right\|\right)\left\|x_{n}-y_{n}\right\| \\
+2 \mu_{2}\left\|B_{2} p-B_{2} x_{n}\right\|\left\|u_{n}-q\right\| \\
+2 \mu_{1}\left\|B_{1} q-B_{1} u_{n}\right\|\left\|v_{n}-p\right\| .
\end{gathered}
$$

From (46), (54), (61), condition (ii), and the boundedness of $\left\{x_{n}\right\},\left\{y_{n}\right\},\left\{u_{n}\right\}$, and $\left\{v_{n}\right\}$, we deduce that

$$
\begin{aligned}
& \lim _{n \rightarrow \infty} \psi_{1}\left(\left\|x_{n}-u_{n}-(p-q)\right\|\right)=0, \\
& \lim _{n \rightarrow \infty} \psi_{2}\left(\left\|u_{n}-v_{n}+(p-q)\right\|\right)=0 .
\end{aligned}
$$

Utilizing the properties of $\psi_{1}$ and $\psi_{2}$, we deduce that

$$
\begin{aligned}
& \lim _{n \rightarrow \infty}\left\|x_{n}-u_{n}-(p-q)\right\|=0, \\
& \lim _{n \rightarrow \infty}\left\|u_{n}-v_{n}+(p-q)\right\|=0 .
\end{aligned}
$$

From (63) we get

$$
\begin{aligned}
& \left\|x_{n}-v_{n}\right\| \\
& \quad \leq\left\|x_{n}-u_{n}-(p-q)\right\|+\left\|u_{n}-v_{n}+(p-q)\right\| \\
& \quad \longrightarrow 0 \text { as } n \longrightarrow \infty,
\end{aligned}
$$

which together with (54), leads to

$$
\begin{aligned}
& \lim _{n \rightarrow \infty}\left\|x_{n}-G x_{n}\right\|=0, \\
& \lim _{n \rightarrow \infty}\left\|x_{n}-S_{n} x_{n}\right\|=0 .
\end{aligned}
$$

Since

$$
\left\|x_{n}-S x_{n}\right\| \leq\left\|x_{n}-S_{n} x_{n}\right\|+\left\|S_{n} x_{n}-S x_{n}\right\| .
$$

Utilizing the assumption on $\left\{S_{n}\right\}$ and Lemma 11, from (65) we get

$$
\lim _{n \rightarrow \infty}\left\|x_{n}-S x_{n}\right\|=0
$$



$\Delta$.

Next, let us show that $\left\{x_{n}\right\}$ converges weakly to some $\bar{x} \epsilon$

Indeed, since $X$ is reflexive and $\left\{x_{n}\right\}$ is bounded, there exists a subsequence $\left\{x_{n_{i}}\right\}$ of $\left\{x_{n}\right\}$ such that $x_{n_{i}}-\bar{x} \in C$. Then by Lemma 6 , we obtain from (44), (65), and (67) that $\bar{x} \in \Gamma$, $\bar{x} \in \operatorname{Fix}(G)=\Omega$, and $\bar{x} \in \operatorname{Fix}(S)=\bigcap_{i=0}^{\infty} \operatorname{Fix}\left(S_{i}\right)$. Thus, $\bar{x} \in \Delta$. In addition, if $\left\{x_{m_{j}}\right\}$ is another subsequence of $\left\{x_{n}\right\}$ such that $x_{m_{j}}-\widehat{x}$, then by Lemma 6 we also deduce from (44), (65),

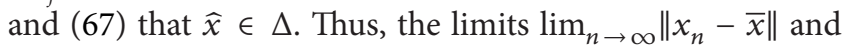
$\lim _{n \rightarrow \infty}\left\|x_{n}-\widehat{x}\right\|$ exist. Now we claim that $\bar{x}=\widehat{x}$. Assume that $\bar{x} \neq \hat{x}$. Then in terms of Opial's condition, we get

$$
\begin{aligned}
\lim _{n \rightarrow \infty}\left\|x_{n}-\bar{x}\right\| & =\limsup _{i \rightarrow \infty}\left\|x_{n_{i}}-\bar{x}\right\|<\limsup _{i \rightarrow \infty}\left\|x_{n_{i}}-\hat{x}\right\| \\
& =\limsup _{n \rightarrow \infty}\left\|x_{n}-\hat{x}\right\|=\limsup _{j \rightarrow \infty}\left\|x_{m_{j}}-\hat{x}\right\| \\
& <\limsup _{j \rightarrow \infty}\left\|x_{m_{j}}-\bar{x}\right\|=\lim _{n \rightarrow \infty}\left\|x_{n}-\bar{x}\right\|,
\end{aligned}
$$

which leads to a contradiction. So, we must have $\bar{x}=\widehat{x}$. Therefore, $x_{n}-\bar{x} \in \Delta$. This completes the proof.

Theorem 15. Let $X$ be a uniformly convex and 2-uniformly smooth Banach space and let $C$ be a nonempty closed convex subset of $X$ such that $C \pm C \subset C$. Let $\Pi_{C}$ be a sunny nonexpansive retraction from $X$ onto $C$. Let $T$ and $F: X \rightarrow$ $C B(X)$ and let $A: C \rightarrow 2^{C}$ be three multivalued mappings, let $g: X \rightarrow C$ be a single-valued mapping, and let $N(\cdot, \cdot)$ : $X \times X \rightarrow C$ be a single-valued continuous mapping satisfying conditions (C1)-(C5) in Theorem 2. Let $B_{i}: C \rightarrow X$ be $\alpha_{i^{-}}$ inverse strongly accretive for $i=1,2$. Let $\left\{S_{i}\right\}_{i=0}^{\infty}$ be a countable family of nonexpansive mappings of $C$ into itself such that $\Delta:=$ $\bigcap_{i=0}^{\infty} \operatorname{Fix}\left(S_{i}\right) \cap \Omega \cap \Gamma \neq \emptyset$, where $\Omega$ is the fixed point set of the mapping $G=\Pi_{C}\left(I-\mu_{1} B_{1}\right) \Pi_{C}\left(I-\mu_{2} B_{2}\right)$ with $0<\mu_{i}<\alpha_{i} / \kappa^{2}$ for $i=1,2$. Let $\left\{\alpha_{n}\right\},\left\{\beta_{n}\right\} \subset[0,1]$, and $\left\{\sigma_{n}\right\} \subset(0, \infty)$ such that $\lim _{n \rightarrow \infty} \alpha_{n} \sigma_{n}=0$ and $\sum_{n=0}^{\infty} \alpha_{n} \sigma_{n}=\infty$. For arbitrary $x_{0} \in C$ define the sequence $\left\{x_{n}\right\}$ iteratively by (32), where $\left\{u_{n}\right\}$ is defined by (33) for any $w_{n} \in T x_{n}, k_{n} \in F x_{n}$ and some $\varepsilon>0$. Then, there exists $\bar{d}>0$ such that for $0<\alpha_{n} \sigma_{n} \leq \bar{d}$, for all $n \geq 0,\left\{x_{n}\right\}$ converges strongly to $p \in \Delta$ provided $\lim _{n \rightarrow \infty}\left\|x_{n+1}-x_{n}\right\|=0$; in this case, for any $w \in T \bar{x}, k \in F \bar{x}$, $(\bar{x}, w, k)$ is a solution of the MVVI (15).

Proof. First of all, repeating the same arguments as those in the proof of Theorem 14, we can prove that for any $v \in C$, $\lambda>0$, there exists a point $\tilde{x} \in D(A)=C$ such that $(\widetilde{x}, w, k)$ is a solution of the MVVI (14), for any $w \in T \widetilde{x}$ and $k \in F \tilde{x}$. In addition, in terms of Proposition 5 we know that $V+\lambda A \circ g$ is a single-valued mapping due to the fact that $V+\lambda A \circ g$ is $\phi$-strongly accretive. Meantime, by Lemma 13 we know that $G: C \rightarrow C$ is nonexpansive.

Without loss of generality we may assume that $v=0$ and $\lambda=1$. Let $p \in \Delta$ and let $r>0$ be sufficiently large such that $x_{0} \in \bar{B}_{r}(p)=: B$. Let $M:=\sup \{\|u\|: u \in N(w, k)+$ $A(g(x)), x \in B, w \in T x, k \in F x\}$. Then as $A \circ g, T$ and $F$ are $H$-uniformly continuous on $X$, for $\varepsilon_{1}:=\phi(r) / 8(1+\varepsilon)$ and $\varepsilon_{2}:=\phi(r) / 8 \mu(1+\varepsilon), \varepsilon_{3}:=\phi(r) / 8 \xi(1+\varepsilon)$, there exist $\delta_{1}, \delta_{2}, \delta_{3}>0$ such that for any $x, y \in X,\|x-y\|<\delta_{1}$, $\|x-y\|<\delta_{2}$, and $\|x-y\|<\delta_{3}$ imply $H(A \circ g(x), A \circ$ $g(y))<\varepsilon_{1}, H(T x, T y)<\varepsilon_{2}$ and $H(F x, F y)<\varepsilon_{3}$, respectively. Let $\bar{d}:=(1 / 2) \min \left\{\delta_{2} / M, \delta_{3} / M, \delta_{1} / M, r / M\right\}$. Taking into account $\lim _{n \rightarrow \infty} \alpha_{n} \sigma_{n}=0$ we may assume that $0<\alpha_{n} \sigma_{n} \leq \bar{d}$, for all $n \geq 0$.

Let us show that $x_{n} \in B$ for all $n \geq 0$. We show this by induction. First, $x_{0} \in B$ by construction. Assume that $x_{n} \in B$. We show that $x_{n+1} \in B$. If possible we assume that $x_{n+1} \notin B$, then $\left\|x_{n+1}-p\right\|>r$. Further from (32) it follows that

$$
\begin{aligned}
\left\|y_{n}-p\right\| & \leq \beta_{n}\left\|S_{n} x_{n}-p\right\|+\left(1-\beta_{n}\right)\left\|G x_{n}-p\right\| \\
& \leq \beta_{n}\left\|x_{n}-p\right\|+\left(1-\beta_{n}\right)\left\|x_{n}-p\right\| \\
& =\left\|x_{n}-p\right\| .
\end{aligned}
$$

Repeating the same arguments as those of (37) in the proof of Theorem 14, we can get

$$
\begin{gathered}
\left\|x_{n+1}-p\right\|^{2} \\
\leq\left\|x_{n}-p\right\|^{2}-2 \alpha_{n} \sigma_{n} \phi\left(\left\|x_{n+1}-p\right\|\right)\left\|x_{n+1}-p\right\| \\
+2 \alpha_{n} \sigma_{n}\left[\left\|N\left(w_{n+1}, k_{n+1}\right)-N\left(w_{n}, k_{n}\right)\right\|\right. \\
\left.+\left\|u_{n+1}-u_{n}\right\|\right]\left\|x_{n+1}-p\right\| \\
\leq\left\|x_{n}-p\right\|^{2}-2 \alpha_{n} \sigma_{n} \phi\left(\left\|x_{n+1}-p\right\|\right)\left\|x_{n+1}-p\right\| \\
+2 \alpha_{n} \sigma_{n}\left[\left\|N\left(w_{n+1}, k_{n+1}\right)-N\left(w_{n+1}, k_{n}\right)\right\|\right. \\
+\left\|N\left(w_{n+1}, k_{n}\right)-N\left(w_{n}, k_{n}\right)\right\| \\
\left.+\left\|u_{n+1}-u_{n}\right\|\right]\left\|x_{n+1}-p\right\| .
\end{gathered}
$$

Utilizing (33) and (70) and repeating the same arguments as those of (40) in the proof of Theorem 14, we can derive $\left\|x_{n+1}-p\right\|^{2} \leq\left\|x_{n}-p\right\|^{2}$. So, we have $\left\|x_{n+1}-p\right\| \leq r$, a contradiction. Therefore, $\left\{x_{n}\right\}$ is bounded.

Next let us show that $x_{n} \rightarrow \bar{x}$ as $n \rightarrow \infty$.

Indeed, since $\left\|x_{n+1}-x_{n}\right\| \rightarrow 0$ as $n \rightarrow \infty$, we have that $H\left(A\left(g\left(x_{n+1}\right)\right), A\left(g\left(x_{n}\right)\right)\right) \rightarrow 0$ and $\| N\left(w_{n+1}, k_{n+1}\right)-$ $N\left(w_{n}, k_{n}\right) \| \rightarrow 0$ as $n \rightarrow \infty$. The conclusion now follows from inequality (70) with the use of Proposition 3 and hence $(\bar{x}, w, k)$ is a solution of the MVVI (15) for any $w \in T \bar{x}$, $k \in F \bar{x}$. This completes the proof.

Remark 16. Theorems 14 and 15 improve, extend, supplement, and develop [4, Theorem 3.2] and [2, Theorem 3.1] in the following aspects.

(i) The problem of finding a point of $\bigcap_{i=0}^{\infty} \operatorname{Fix}\left(S_{i}\right) \cap \Omega \cap \Gamma$ in Theorems 14 and 15 is more general and more subtle than every one of the problem of finding a point of $\Gamma$ in $[4$, Theorem 3.2] and the problem of finding a point of $\bigcap_{i=1}^{\infty} \operatorname{Fix}\left(S_{i}\right) \cap \Omega$ in [2, Theorem 3.1].

(ii) The iterative scheme in [2, Theorem 3.1] is extended to develop the iterative scheme (32) of Theorems 14 and 15 by virtue of the iterative schemes of $[4$, Theorems 3.2]. The iterative scheme (32) of Theorems 14 and 15 is more advantageous and more flexible than the 
iterative scheme of [4, Theorem 3.2] because it can be applied to solving three problems (i.e., GSVI (9), MVVI (15), and the fixed point problem of $\left.\left\{S_{n}\right\}\right)$ and involves several parameter sequences $\left\{\alpha_{n}\right\},\left\{\beta_{n}\right\}$, and $\left\{\sigma_{n}\right\}$.

(iii) Theorems 14 and 15 extend and generalize [4, Theorems 3.2] to the setting of a countable family of nonexpansive mappings and the GSVI (9) for two inverse-strongly accretive mappings. In the meantime, Theorems 14 and 15 extend and generalize [2, Theorem 3.1] to the setting of the MVVI (15).

(iv) The iterative scheme (32) in Theorems 14 and 15 is very different from everyone in [4, Theorem 3.2] and [2, Theorem 3.1] because the iterative scheme in [2, Theorem 3.1] involves the viscosity approximation method and the iterative scheme in [4, Theorem 3.2] is a one-step iterative scheme.

(v) No boundedness condition on the ranges $R(I-$ $N(T(\cdot), F(\cdot)))$ and $R(A(g(\cdot)))$ is imposed in Theorems 14 and 15.

\section{Mann-Type Extragradient Algorithms in Smooth and Uniformly Convex Banach Spaces}

In this section, we introduce Mann-type extragradient algorithms in smooth and uniformly convex Banach spaces and show weak and strong convergence theorems. First, we give some useful lemmas whose proofs will be omitted.

Lemma 17. Let $C$ be a nonempty closed convex subset of a smooth Banach space $X$ and let the mapping $B_{i}: C \rightarrow X$ be $\lambda_{i}$-strictly pseudocontractive and $\alpha_{i}$-strongly accretive with $\alpha_{i}+\lambda_{i} \geq 1$ for $i=1,2$. Then, for $\mu_{i} \in(0,1]$ we have

$$
\begin{aligned}
& \left\|\left(I-\mu_{i} B_{i}\right) x-\left(I-\mu_{i} B_{i}\right) y\right\| \\
& \leq\left\{\sqrt{\frac{1-\alpha_{i}}{\lambda_{i}}}+\left(1-\mu_{i}\right)\left(1+\frac{1}{\lambda_{i}}\right)\right\} \\
& \quad \times\|x-y\|, \quad \forall x, y \in C,
\end{aligned}
$$

for $i=1$, 2. In particular, if $1-\left(\lambda_{i} /\left(1+\lambda_{i}\right)\right)\left(1-\sqrt{\left(1-\alpha_{i}\right) / \lambda_{i}}\right) \leq$ $\mu_{i} \leq 1$, then $I-\mu_{i} B_{i}$ is nonexpansive for $i=1,2$.

Lemma 18. Let $C$ be a nonempty closed convex subset of a smooth Banach space $X$. Let $\Pi_{C}$ be a sunny nonexpansive retraction from $X$ onto $C$ and let the mapping $B_{i}: C \rightarrow X$ be $\lambda_{i}$-strictly pseudocontractive and $\alpha_{i}$-strongly accretive with $\alpha_{i}+$ $\lambda_{i} \geq 1$ for $i=1,2$. Let $G: C \rightarrow C$ be the mapping defined by

$$
\begin{aligned}
G(x)=\Pi_{C} & {\left[\Pi_{C}\left(x-\mu_{2} B_{2} x\right)\right.} \\
& \left.-\mu_{1} B_{1} \Pi_{C}\left(x-\mu_{2} B_{2} x\right)\right], \forall x \in C .
\end{aligned}
$$

If $1-\left(\lambda_{i} /\left(1+\lambda_{i}\right)\right)\left(1-\sqrt{\left(1-\alpha_{i}\right) / \lambda_{i}}\right) \leq \mu_{i} \leq 1$, then $G: C \rightarrow C$ is nonexpansive.
Theorem 19. Let $X$ be a smooth and uniformly convex Banach space satisfying Opial's condition and $C$ be a nonempty closed convex subset of $X$ such that $C \pm C \subset C$. Let $\Pi_{C}$ be a sunny nonexpansive retraction from $X$ onto $C$. Let $T$ and $F: X \rightarrow$ $C B(X)$ and let $A: C \rightarrow 2^{C}$ be three multivalued mappings, let $g: X \rightarrow C$ be a single-valued mapping, and let $N(\cdot, \cdot)$ : $X \times X \rightarrow C$ be a single-valued continuous mapping satisfying conditions $(C 1)-(C 5)$ in Theorem 2 and

(H6) $N(T x, F x)+A(g(x)): X \rightarrow C$ is $\lambda_{0}$-strictly pseudocontractive and $\alpha_{0}$-strongly accretive with $\lambda_{0}+\alpha_{0} \geq 1$.

Let $B_{i}: C \rightarrow X$ be $\lambda_{i}$-strictly pseudocontractive and $\alpha_{i}$-strongly accretive with $\lambda_{i}+\alpha_{i} \geq 1$ for $i=1,2$. Let $\left\{S_{i}\right\}_{i=0}^{\infty}$ be a countable family of nonexpansive mappings of $C$ into itself such that $\Delta:=$ $\bigcap_{i=0}^{\infty} \operatorname{Fix}\left(S_{i}\right) \cap \Omega \cap \Gamma \neq \emptyset$, where $\Omega$ is the fixed point set of the mapping $G=\Pi_{C}\left(I-\mu_{1} B_{1}\right) \Pi_{C}\left(I-\mu_{2} B_{2}\right)$ with $1-\left(\lambda_{i} /(1+\right.$ $\left.\left.\lambda_{i}\right)\right)\left(1-\sqrt{\left(1-\alpha_{i}\right) / \lambda_{i}}\right) \leq \mu_{i} \leq 1$ for $i=1,2$. Assume that $\left\{\alpha_{n}\right\}$, $\left\{\beta_{n}\right\},\left\{\gamma_{n}\right\}$, and $\left\{\sigma_{n}\right\}$ are sequences in $[0,1]$ such that

(i) $0<\liminf _{n \rightarrow \infty} \alpha_{n} \leq \limsup _{n \rightarrow \infty} \alpha_{n}<1$;

(ii) $\left\{\beta_{n}\right\},\left\{\gamma_{n}\right\},\left\{\beta_{n}+\gamma_{n}\right\} \subset[c, d]$ for some $c, d \in(0,1)$;

(iii) $0<\liminf _{n \rightarrow \infty} \sigma_{n} \leq \limsup _{n \rightarrow \infty} \sigma_{n}<1$.

For arbitrary $x_{0} \in C$ define the sequence $\left\{x_{n}\right\}$ iteratively by

$$
\begin{aligned}
y_{n}= & \beta_{n} S_{n} x_{n}+\gamma_{n} x_{n}+\left(1-\beta_{n}-\gamma_{n}\right) \Pi_{C}\left(I-\mu_{1} B_{1}\right) \\
& \times \Pi_{C}\left(I-\mu_{2} B_{2}\right) x_{n}, \\
x_{n+1}= & \alpha_{n}\left[x_{n}-\sigma_{n}\left(N\left(w_{n}, k_{n}\right)+u_{n}\right)\right] \\
& +\left(1-\alpha_{n}\right) y_{n}, \quad u_{n} \in A\left(g\left(x_{n}\right)\right), \quad \forall n \geq 0,
\end{aligned}
$$

where $\left\{u_{n}\right\}$ is defined by

$$
\begin{array}{r}
\left\|u_{n}-u_{n+1}\right\| \leq(1+\varepsilon) H\left(A\left(g\left(x_{n+1}\right)\right), A\left(g\left(x_{n}\right)\right)\right), \\
\forall n \geq 0,
\end{array}
$$

for any $w_{n} \in T x_{n}, k_{n} \in F x_{n}$ and some $\varepsilon>0$. Assume that $\sum_{n=0}^{\infty} \sup _{x \in D}\left\|S_{n+1} x-S_{n} x\right\|<\infty$ for any bounded subset $D$ of $C$ and let $S$ be a mapping of $C$ into itself defined by $S x=\lim _{n \rightarrow \infty} S_{n} x$ for all $x \in C$ and suppose that $\operatorname{Fix}(S)=$ $\bigcap_{i=0}^{\infty} \operatorname{Fix}\left(S_{i}\right)$. Then $\left\{x_{n}\right\}$ converges weakly to some $\bar{x} \in \Delta$, and for any $w \in T \bar{x}, k \in F \bar{x},(\bar{x}, w, k)$ is a solution of the MVVI (15).

Proof. First of all, repeating the same arguments as those in the proof of Theorem 14, we can prove that for any $v \in C, \lambda>$ 0 , there exists a point $\tilde{x} \in C$ such that $(\tilde{x}, w, k)$ is a solution of the MVVI (14), for any $w \in T \tilde{x}$ and $k \in F \tilde{x}$. In addition, in terms of Proposition 5 we know that $V+\lambda A \circ g$ is a singlevalued mapping due to the fact that $V+\lambda A \circ g$ is $\phi$-strongly accretive. Assume that $N(T x, F x)+\lambda A(g(x)): X \rightarrow C$ is $\lambda_{0}$ strictly pseudocontractive and $\alpha_{0}$ strongly accretive with $\lambda_{0}+\alpha_{0} \geq 1$. Then by Lemma 17 we conclude that the mapping $x \mapsto x-(N(T x, F x)+\lambda A(g(x)))$ is nonexpansive. Meantime, by Lemma 18 we know that $G: C \rightarrow C$ is also nonexpansive. 
Without loss of generality we may assume that $v=0$ and $\lambda=1$. Let $p \in \Delta$ and let $r>0$ be sufficiently large such that $x_{0} \in \bar{B}_{r}(p)=: B$. Observe that

$$
\begin{aligned}
\left\|y_{n}-p\right\| \leq & \beta_{n}\left\|S_{n} x_{n}-p\right\|+\gamma_{n}\left\|x_{n}-p\right\| \\
& +\left(1-\beta_{n}-\gamma_{n}\right)\left\|G x_{n}-p\right\| \\
\leq & \beta_{n}\left\|x_{n}-p\right\|+\gamma_{n}\left\|x_{n}-p\right\| \\
& +\left(1-\beta_{n}-\gamma_{n}\right)\left\|x_{n}-p\right\| \\
= & \left\|x_{n}-p\right\| .
\end{aligned}
$$

Utilizing (75) and repeating the same arguments as those in the proof of Theorem 14, we can derive $x_{n} \in B$ for all $n \geq 0$. Hence $\left\{x_{n}\right\}$ is bounded.

Let us show that $\lim _{n \rightarrow \infty}\left\|x_{n}-y_{n}\right\|=0$ and $\lim _{n \rightarrow \infty} \| x_{n}-$ $x_{n+1} \|=0$.

Indeed, repeating the same arguments as those of (41) in the proof of Theorem 14, we obtain from (73) and (75) that for all $n \geq 0$

$$
\begin{aligned}
\left\|x_{n+1}-p\right\|^{2} \leq & \left\|x_{n}-p\right\|^{2}-\alpha_{n} \sigma_{n}\left(1-\sigma_{n}\right) \varphi_{1} \\
& \times\left(\left\|N\left(w_{n}, k_{n}\right)+u_{n}\right\|\right)-\alpha_{n}\left(1-\alpha_{n}\right) \varphi \\
& \times\left(\left\|x_{n}-y_{n}-\sigma_{n}\left(N\left(w_{n}, k_{n}\right)+u_{n}\right)\right\|\right) \\
\leq & \left\|x_{n}-p\right\|^{2} .
\end{aligned}
$$

It is easy to see that the limit $\lim _{n \rightarrow \infty}\left\|x_{n}-p\right\|$ exists. Meantime, it can be readily seen from (76) that

$$
\begin{aligned}
& \alpha_{n} \sigma_{n}\left(1-\sigma_{n}\right) \varphi_{1}\left(\left\|N\left(w_{n}, k_{n}\right)+u_{n}\right\|\right) \\
& \quad+\alpha_{n}\left(1-\alpha_{n}\right) \varphi\left(\left\|x_{n}-y_{n}-\sigma_{n}\left(N\left(w_{n}, k_{n}\right)+u_{n}\right)\right\|\right) \\
& \leq\left\|x_{n}-p\right\|^{2}-\left\|x_{n+1}-p\right\|^{2},
\end{aligned}
$$

which together with conditions (i), (iii), and the existence of $\lim _{n \rightarrow \infty}\left\|x_{n}-p\right\|$, implies that

$$
\begin{gathered}
\lim _{n \rightarrow \infty} \varphi_{1}\left(\left\|N\left(w_{n}, k_{n}\right)+u_{n}\right\|\right)=0, \\
\lim _{n \rightarrow \infty} \varphi\left(\left\|x_{n}-y_{n}-\sigma_{n}\left(N\left(w_{n}, k_{n}\right)+u_{n}\right)\right\|\right)=0 .
\end{gathered}
$$

Utilizing the properties of $\varphi$ and $\varphi_{1}$, we get

$$
\begin{gathered}
\operatorname{Lim}_{n \rightarrow \infty}\left\|N\left(w_{n}, k_{n}\right)+u_{n}\right\|=0, \\
\lim _{n \rightarrow \infty}\left\|x_{n}-y_{n}-\sigma_{n}\left(N\left(w_{n}, k_{n}\right)+u_{n}\right)\right\|=0 .
\end{gathered}
$$

Note that

$$
\begin{aligned}
\left\|x_{n}-y_{n}\right\| \leq & \left\|x_{n}-y_{n}-\sigma_{n}\left(N\left(w_{n}, k_{n}\right)+u_{n}\right)\right\| \\
& +\sigma_{n}\left\|N\left(w_{n}, k_{n}\right)+u_{n}\right\| .
\end{aligned}
$$

So, we have

$$
\lim _{n \rightarrow \infty}\left\|x_{n}-y_{n}\right\|=0
$$

Also, observe that

$$
\begin{aligned}
\left\|x_{n+1}-x_{n}\right\| \leq & \alpha_{n} \sigma_{n}\left\|N\left(w_{n}, k_{n}\right)+u_{n}\right\| \\
& +\left(1-\alpha_{n}\right)\left\|x_{n}-y_{n}\right\| \\
\leq & \left\|N\left(w_{n}, k_{n}\right)+u_{n}\right\|+\left\|x_{n}-y_{n}\right\| .
\end{aligned}
$$

Thus from (79) and (81) it follows that

$$
\lim _{n \rightarrow \infty}\left\|x_{n+1}-x_{n}\right\|=0 .
$$

Let us show that $\lim _{n \rightarrow \infty}\left\|x_{n}-G x_{n}\right\|=\lim _{n \rightarrow \infty} \| x_{n}-$ $S x_{n} \|=0$.

Indeed, from (73) we obtain that

$$
\begin{aligned}
& \frac{\beta_{n}}{\beta_{n}+\gamma_{n}}\left(S_{n} x_{n}-G x_{n}\right)+\frac{\gamma_{n}}{\beta_{n}+\gamma_{n}}\left(x_{n}-G x_{n}\right) \\
& =\frac{\beta_{n} S_{n} x_{n}+\gamma_{n} x_{n}}{\beta_{n}+\gamma_{n}}-G x_{n} \\
& =\frac{y_{n}-\left(1-\beta_{n}-\gamma_{n}\right) G x_{n}}{\beta_{n}+\gamma_{n}}-G x_{n} \\
& =\frac{y_{n}-G x_{n}}{\beta_{n}+\gamma_{n}} .
\end{aligned}
$$

Utilizing Lemma 10 we deduce from (73) and (84) that

$$
\begin{gathered}
\left\|y_{n}-p\right\|^{2} \\
=\| \beta_{n}\left(S_{n} x_{n}-p\right)+\gamma_{n}\left(x_{n}-p\right) \\
+\left(1-\beta_{n}-\gamma_{n}\right)\left(G x_{n}-p\right) \|^{2} \\
=\|\left(\beta_{n}+\gamma_{n}\right)\left[\frac{\beta_{n}}{\beta_{n}+\gamma_{n}}\left(S_{n} x_{n}-p\right)\right. \\
\left.+\frac{\gamma_{n}}{\beta_{n}+\gamma_{n}}\left(x_{n}-p\right)\right] \\
+\left(1-\beta_{n}-\gamma_{n}\right)\left(G x_{n}-p\right) \|^{2} \\
\leq\left(\beta_{n}+\gamma_{n}\right) \| \frac{\beta_{n}}{\beta_{n}+\gamma_{n}}\left(S_{n} x_{n}-p\right) \\
+\frac{\gamma_{n}}{\beta_{n}+\gamma_{n}}\left(x_{n}-p\right) \|^{2} \\
+\left(1-\beta_{n}-\gamma_{n}\right)\left\|G x_{n}-p\right\|^{2} \\
-\left(\beta_{n}+\gamma_{n}\right)\left(1-\beta_{n}-\gamma_{n}\right) \varphi_{2} \\
\times\left(\| \frac{\beta_{n}}{\beta_{n}+\gamma_{n}}\left(S_{n} x_{n}-G x_{n}\right)\right. \\
\left.+\frac{\gamma_{n}}{\beta_{n}+\gamma_{n}}\left(x_{n}-G x_{n}\right) \|\right)
\end{gathered}
$$




$$
\begin{aligned}
& =\left(\beta_{n}+\gamma_{n}\right) \| \frac{\beta_{n}}{\beta_{n}+\gamma_{n}}\left(S_{n} x_{n}-p\right) \\
& +\frac{\gamma_{n}}{\beta_{n}+\gamma_{n}}\left(x_{n}-p\right) \|^{2} \\
& +\left(1-\beta_{n}-\gamma_{n}\right)\left\|G x_{n}-p\right\|^{2} \\
& -\left(\beta_{n}+\gamma_{n}\right)\left(1-\beta_{n}-\gamma_{n}\right) \varphi_{2} \\
& \times\left(\frac{1}{\beta_{n}+\gamma_{n}}\left\|y_{n}-G x_{n}\right\|\right) \\
& \leq\left(\beta_{n}+\gamma_{n}\right)\left[\frac{\beta_{n}}{\beta_{n}+\gamma_{n}}\left\|S_{n} x_{n}-p\right\|^{2}\right. \\
& +\frac{\gamma_{n}}{\beta_{n}+\gamma_{n}}\left\|x_{n}-p\right\|^{2} \\
& \left.-\frac{\beta_{n} \gamma_{n}}{\left(\beta_{n}+\gamma_{n}\right)^{2}} \varphi_{3}\left(\left\|S_{n} x_{n}-x_{n}\right\|\right)\right] \\
& +\left(1-\beta_{n}-\gamma_{n}\right)\left\|G x_{n}-p\right\|^{2} \\
& -\left(\beta_{n}+\gamma_{n}\right)\left(1-\beta_{n}-\gamma_{n}\right) \varphi_{2} \\
& \times\left(\frac{1}{\beta_{n}+\gamma_{n}}\left\|y_{n}-G x_{n}\right\|\right) \\
& \leq \beta_{n}\left\|S_{n} x_{n}-p\right\|^{2}+\gamma_{n}\left\|x_{n}-p\right\|^{2} \\
& -\beta_{n} \gamma_{n} \varphi_{3}\left(\left\|S_{n} x_{n}-x_{n}\right\|\right) \\
& +\left(1-\beta_{n}-\gamma_{n}\right)\left\|G x_{n}-p\right\|^{2} \\
& -\left(\beta_{n}+\gamma_{n}\right)\left(1-\beta_{n}-\gamma_{n}\right) \varphi_{2} \\
& \times\left(\frac{1}{\beta_{n}+\gamma_{n}}\left\|y_{n}-G x_{n}\right\|\right) \\
& \leq \beta_{n}\left\|x_{n}-p\right\|^{2}+\gamma_{n}\left\|x_{n}-p\right\|^{2} \\
& -\beta_{n} \gamma_{n} \varphi_{3}\left(\left\|S_{n} x_{n}-x_{n}\right\|\right) \\
& +\left(1-\beta_{n}-\gamma_{n}\right)\left\|x_{n}-p\right\|^{2} \\
& -\left(\beta_{n}+\gamma_{n}\right)\left(1-\beta_{n}-\gamma_{n}\right) \varphi_{2} \\
& \times\left(\frac{1}{\beta_{n}+\gamma_{n}}\left\|y_{n}-G x_{n}\right\|\right) \\
& =\left\|x_{n}-p\right\|^{2}-\beta_{n} \gamma_{n} \varphi_{3}\left(\left\|S_{n} x_{n}-x_{n}\right\|\right) \\
& -\left(\beta_{n}+\gamma_{n}\right)\left(1-\beta_{n}-\gamma_{n}\right) \varphi_{2} \\
& \times\left(\frac{1}{\beta_{n}+\gamma_{n}}\left\|y_{n}-G x_{n}\right\|\right) \text {, }
\end{aligned}
$$

which hence implies that

$$
\begin{aligned}
\beta_{n} \gamma_{n} \varphi_{3} & \left(\left\|S_{n} x_{n}-x_{n}\right\|\right) \\
& +\left(\beta_{n}+\gamma_{n}\right)\left(1-\beta_{n}-\gamma_{n}\right) \varphi_{2} \\
& \times\left(\frac{1}{\beta_{n}+\gamma_{n}}\left\|y_{n}-G x_{n}\right\|\right) \\
\leq & \left\|x_{n}-p\right\|^{2}-\left\|y_{n}-p\right\|^{2} \\
\leq & \left(\left\|x_{n}-p\right\|+\left\|y_{n}-p\right\|\right)\left\|x_{n}-y_{n}\right\| .
\end{aligned}
$$

Utilizing condition (ii) we conclude from (81) and (86) that

$$
\begin{gathered}
\lim _{n \rightarrow \infty} \varphi_{3}\left(\left\|S_{n} x_{n}-x_{n}\right\|\right)=0, \\
\lim _{n \rightarrow \infty} \varphi_{2}\left(\frac{1}{\beta_{n}+\gamma_{n}}\left\|y_{n}-G x_{n}\right\|\right)=0 .
\end{gathered}
$$

From the properties of $\varphi_{2}$ and $\varphi_{3}$ and condition (ii), it immediately follows that

$$
\lim _{n \rightarrow \infty}\left\|S_{n} x_{n}-x_{n}\right\|=0, \quad \lim _{n \rightarrow \infty}\left\|y_{n}-G x_{n}\right\|=0 .
$$

Observe that

$$
\begin{gathered}
\left\|x_{n}-S x_{n}\right\| \leq\left\|x_{n}-S_{n} x_{n}\right\|+\left\|S_{n} x_{n}-S x_{n}\right\|, \\
\left\|x_{n}-G x_{n}\right\| \leq\left\|x_{n}-y_{n}\right\|+\left\|y_{n}-G x_{n}\right\| .
\end{gathered}
$$

Thus, utilizing Lemma 11 and the assumption on $\left\{S_{n}\right\}$, from (81) and (88) we conclude that

$$
\lim _{n \rightarrow \infty}\left\|x_{n}-S x_{n}\right\|=0, \quad \lim _{n \rightarrow \infty}\left\|x_{n}-G x_{n}\right\|=0 .
$$

Next, let us show that $\left\{x_{n}\right\}$ converges weakly to some $\bar{x} \in$ $\Delta$.

Indeed, repeating the same arguments as those in the proof of Theorem 14, we can prove that $\left\{x_{n}\right\}$ converges weakly to some $\bar{x} \in \Delta$. This completes the proof.

Theorem 20. Let $X$ be a uniformly convex and 2-uniformly smooth Banach space and let $C$ be a nonempty closed convex subset of $X$ such that $C \pm C \subset C$. Let $\Pi_{C}$ be a sunny nonexpansive retraction from $X$ onto $C$. Let $T$ and $F: X \rightarrow C B(X)$ and let $A: C \rightarrow 2^{C}$ be three multivalued mappings, let $g: X \rightarrow C$ be a single-valued mapping, and let $N(\cdot, \cdot): X \times X \rightarrow C$ be a single-valued continuous mapping satisfying conditions (C1)-(C5) in Theorem 2. Let $B_{i}: C \rightarrow X$ be $\lambda_{i}$ strictly pseudocontractive and $\alpha_{i}$ strongly accretive with $\lambda_{i}+\alpha_{i} \geq 1$ for $i=1,2$. Let $\left\{S_{i}\right\}_{i=0}^{\infty}$ be a countable family of nonexpansive mappings of $C$ into itself such that $\Delta:=\bigcap_{i=0}^{\infty} \operatorname{Fix}\left(S_{i}\right) \cap \Omega \cap \Gamma \neq \emptyset$, where $\Omega$ is the fixed point set of the mapping $G=\Pi_{C}\left(I-\mu_{1} B_{1}\right) \Pi_{C}\left(I-\mu_{2} B_{2}\right)$ with $1-\left(\lambda_{i} /\left(1+\lambda_{i}\right)\right)\left(1-\sqrt{\left(1-\alpha_{i}\right) / \lambda_{i}}\right) \leq \mu_{i} \leq 1$ for $i=1$, 2. Let $\left\{\alpha_{n}\right\},\left\{\beta_{n}\right\} \subset[0,1]$ and $\left\{\sigma_{n}\right\} \subset(0, \infty)$ such that $\lim _{n \rightarrow \infty} \alpha_{n} \sigma_{n}=$ 0 and $\sum_{n=0}^{\infty} \alpha_{n} \sigma_{n}=\infty$. For arbitrary $x_{0} \in C$ define the sequence $\left\{x_{n}\right\}$ iteratively by (73), where $\left\{u_{n}\right\}$ is defined by (74) for any $w_{n} \in T x_{n}, k_{n} \in F x_{n}$ and some $\varepsilon>0$. Then, there exists $\bar{d}>0$ such that for $0<\alpha_{n} \sigma_{n} \leq \bar{d}$, for all $n \geq 0,\left\{x_{n}\right\}$ converges strongly to $p \in \Delta$ provided $\lim _{n \rightarrow \infty}\left\|x_{n+1}-x_{n}\right\|=0$; in this case, for any $w \in T \bar{x}, k \in F \bar{x},(\bar{x}, w, k)$ is a solution of the MVVI (15). 
Proof. First of all, repeating the same arguments as those in the proof of Theorem 14, we can prove that for any $v \in C, \lambda>$ 0 , there exists a point $\tilde{x} \in D(A)=C$ such that $(\tilde{x}, w, k)$ is a solution of the MVVI (14), for any $w \in T \tilde{x}$ and $k \in F \tilde{x}$. In addition, in terms of Proposition 5 we know that $V+\lambda A \circ g$ is a single-valued mapping due to the fact that $V+\lambda A \circ g$ is $\phi$-strongly accretive. Meantime, by Lemma 18 we know that $G: C \rightarrow C$ is nonexpansive.

Without loss of generality we may assume that $v=0$ and $\lambda=1$. Let $p \in \Delta$. Then from (73) we deduce that for all $n \geq 0$

$$
\begin{aligned}
\left\|y_{n}-p\right\| \leq & \beta_{n}\left\|S_{n} x_{n}-p\right\|+\gamma_{n}\left\|x_{n}-p\right\| \\
& +\left(1-\beta_{n}-\gamma_{n}\right)\left\|G x_{n}-p\right\| \\
\leq & \beta_{n}\left\|x_{n}-p\right\|+\gamma_{n}\left\|x_{n}-p\right\| \\
& +\left(1-\beta_{n}-\gamma_{n}\right)\left\|x_{n}-p\right\| \\
= & \left\|x_{n}-p\right\| .
\end{aligned}
$$

Repeating the same arguments as those of the remainder in the proof of Theorem 15, we can derive the desired result.

\section{Conflict of Interests}

The authors declare that there is no conflict of interests regarding the publication of this paper.

\section{Acknowledgments}

This research was partially supported to the first author by the National Science Foundation of China (11071169), Innovation Program of Shanghai Municipal Education Commission (09ZZ133), and Ph.D. Program Foundation of Ministry of Education of China (20123127110002). This paper was funded by the Deanship of Scientific Research (DSR), King Abdulaziz University, Jeddah. The second and third authors, therefore, acknowledge with thanks DSR for technical and financial support. The authors are also thankful to the referees for their valuable suggestion/comments and appreciation.

\section{References}

[1] Y. Takahashi, K. Hashimoto, and M. Kato, "On sharp uniform convexity, smoothness, and strong type, cotype inequalities," Journal of Nonlinear Convex Analysis, vol. 3, no. 2, pp. 267-281, 2002.

[2] G. Cai and S.Q. Bu, "Convergence analysis for variational inequality problems and fixed point problems in 2-uniformly smooth and uniformly convex banach spaces," Mathematical and Computer Modelling, vol. 55, no. 3-4, pp. 538-546, 2012.

[3] L.-C. Ceng, C.-Y. Wang, and J.-C. Yao, "Strong convergence theorems by a relaxed extragradient method for a general system of variational inequalities," Mathematical Methods of Operations Research, vol. 67, no. 3, pp. 375-390, 2008.

[4] C. E. Chidume, H. Zegeye, and K. R. Kazmi, "Existence and convergence theorems for a class of multi-valued variational inclusions in Banach spaces," Nonlinear Analysis: Theory, Methods \& Applications, vol. 59, no. 5, pp. 649-656, 2004.
[5] Y. Yao, Y.-C. Liou, S. M. Kang, and Y. Yu, "Algorithms with strong convergence for a system of nonlinear variational inequalities in banach spaces," Nonlinear Analysis: Theory, Methods \& Applications, vol. 74, no. 17, pp. 6024-6034, 2011.

[6] L.-C. Ceng, Q. H. Ansari, and J.-C. Yao, "Relaxed extragradient iterative methods for variational inequalities," Applied Mathematics and Computation, vol. 218, no. 3, pp. 1112-1123, 2011.

[7] L.-C. Ceng, Q. H. Ansari, N.-C. Wong, and J.-C. Yao, "An extragradient-like approximation method for variational inequalities and fixed point problems," Fixed Point Theory and Applications, vol. 2011, article 22, 2011.

[8] L.-C. Ceng, S.-M. Guu, and J.-C. Yao, "Finding common solutions of a variational inequality, a general system of variational inequalities, and a fixed-point problem via a hybrid extragradient method," Fixed Point Theory and Application, vol. 2011, Article ID 626159, 22 pages, 2011.

[9] L.-C. Zeng and J.-C. Yao, "Strong convergence theorem by an extragradient method for fixed point problems and variational inequality problems," Taiwanese Journal of Mathematics, vol. 10, no. 5, pp. 1293-1303, 2006.

[10] L.-C. Ceng, H.-K. Xu, and J.-C. Yao, "Strong convergence of an iterative method with perturbed mappings for nonexpansive and accretive operators," Numerical Functional Analysis and Optimization, vol. 29, no. 3-4, pp. 324-345, 2008.

[11] C. E. Chidume and H. Zegeye, "Approximation methods for nonlinear operator equations," Proceedings of the American Mathematical Society, vol. 131, no. 8, pp. 2467-2478, 2003.

[12] S. S. Chang, J. K. Kim, and K. H. Kim, "On the existence and iterative approximation problems of solutions for set-valued variational inclusions in Banach spaces," Journal of Mathematical Analysis and Applications, vol. 268, no. 1, pp. 89-108, 2002.

[13] X. He, "On $\phi$-strongly accretive mappings and some set-valued variational problems," Journal of Mathematical Analysis and Applications, vol. 277, no. 2, pp. 504-511, 2003.

[14] J. S. Jung, "Iterative approaches to common fixed points of nonexpansive mappings in Banach spaces," Journal of Mathematical Analysis and Applications, vol. 302, no. 2, pp. 509-520, 2005.

[15] S. Reich, "Weak convergence theorems for nonexpansive mappings in Banach spaces," Journal of Mathematical Analysis and Applications, vol. 67, no. 2, pp. 274-276, 1979.

[16] H.-K. Xu, "Inequalities in banach spaces with applications," Nonlinear Analysis: Theory, Methods \& Applications, vol. 16, no. 12, pp. 1127-1138, 1991.

[17] K. Aoyama, Y. Kimura, W. Takahashi, and M. Toyoda, "Approximation of common fixed points of a countable family of nonexpansive mappings in a banach space," Nonlinear Analysis: Theory, Methods ef Applications, vol. 67, no. 8, pp. 2350-2360, 2007.

[18] I. Cioranescu, Geometry of Banach Spaces, Duality Mapping and Nonlinear Problems, Kluwer Academic Publishers, Amsterdam, The Netherlands, 1990.

[19] C. H. Morales, "Surjective theorems for multi-valued mappings of accretive type," Commentations Mathematicae Universitatis Carolinae, vol. 26, no. 2, pp. 397-413, 1985.

[20] S. B. Nadler Jr., "Multi-valued contraction mappings," Pacific Journal of Mathematics, vol. 30, no. 2, pp. 475-488, 1969.

[21] S. Kamimura and W. Takahashi, "Strong convergence of a proximal-type algorithm in a Banach space," SIAM Journal on Optimization, vol. 13, no. 3, pp. 938-945, 2006. 


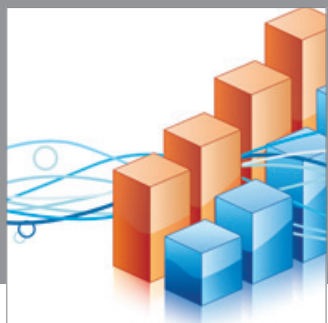

Advances in

Operations Research

mansans

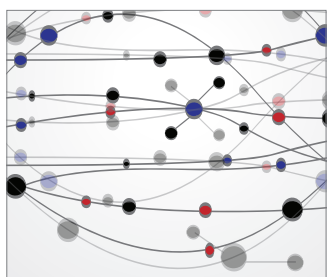

The Scientific World Journal
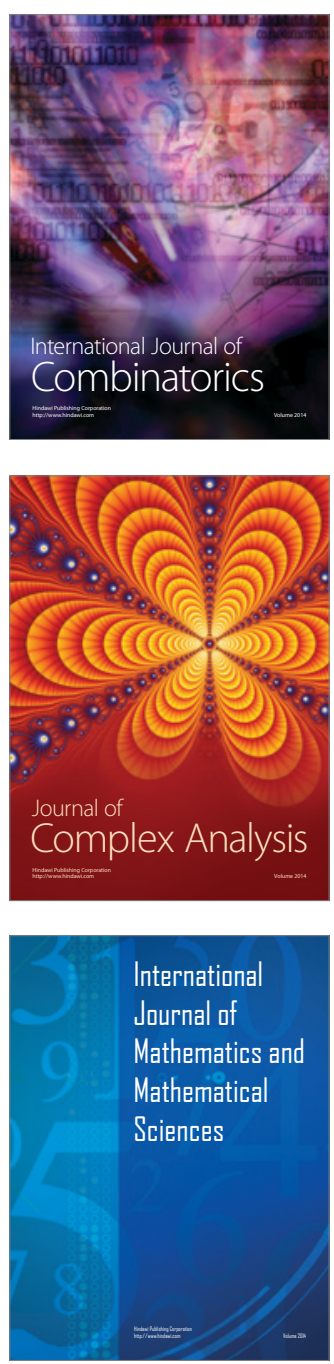
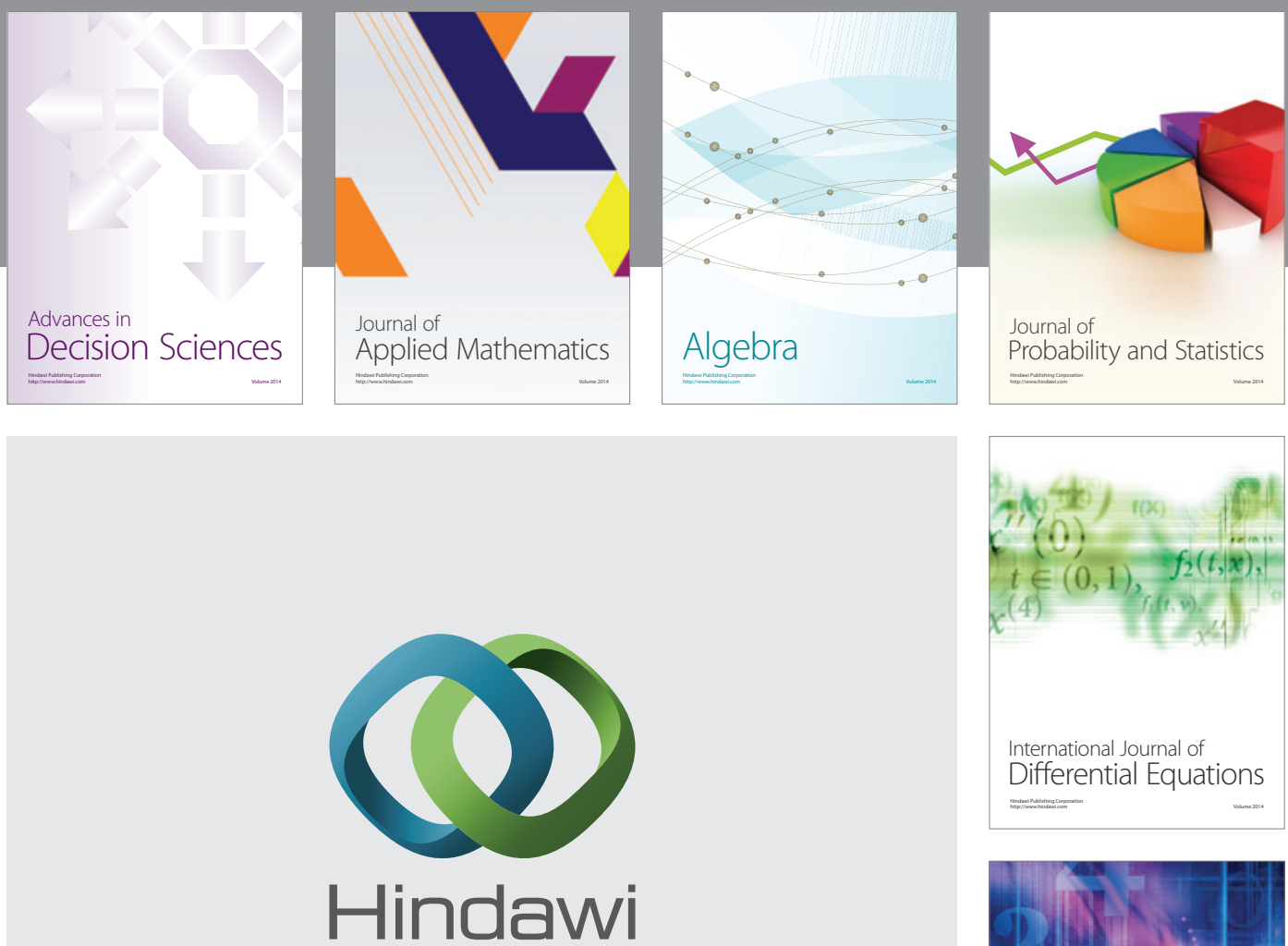

Submit your manuscripts at http://www.hindawi.com
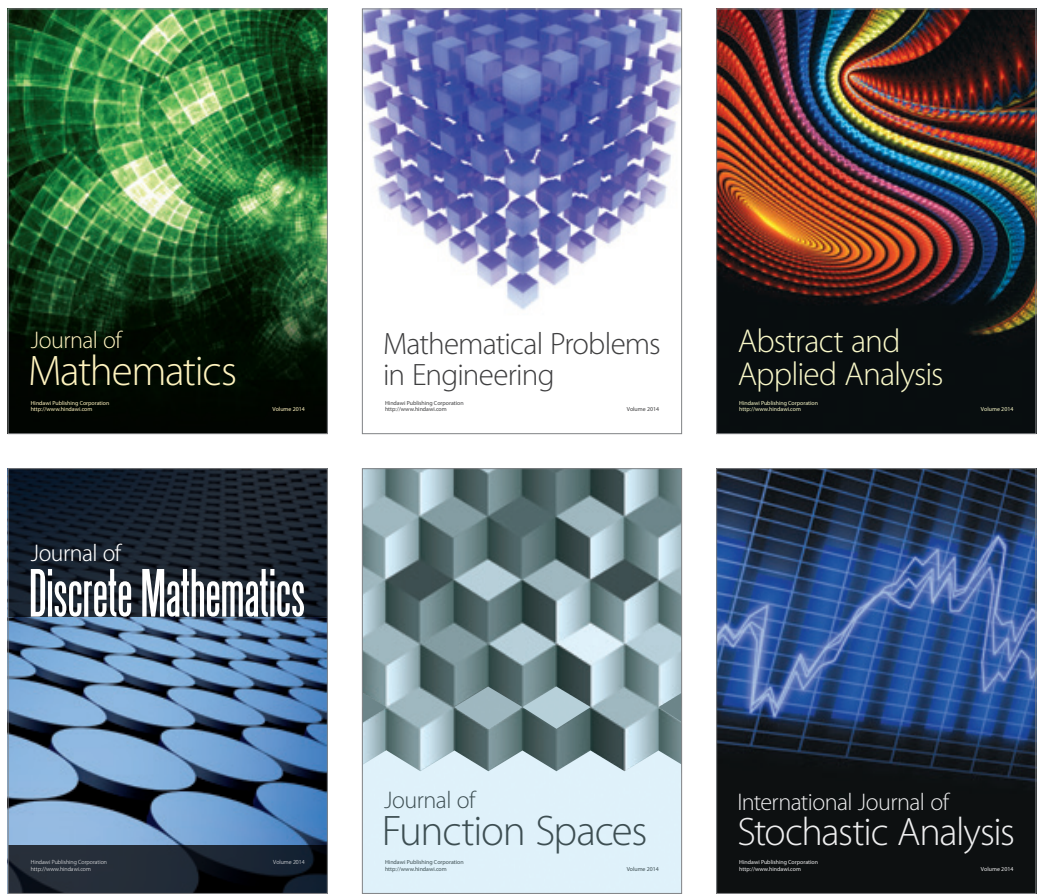

Journal of

Function Spaces

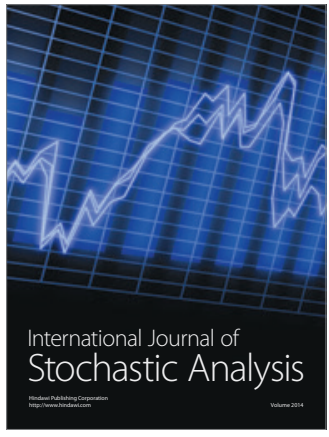

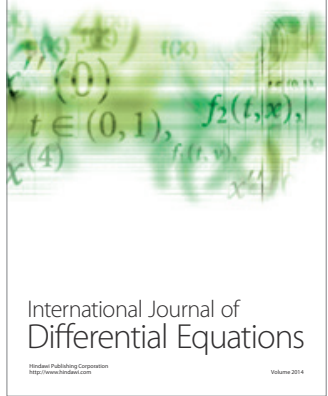
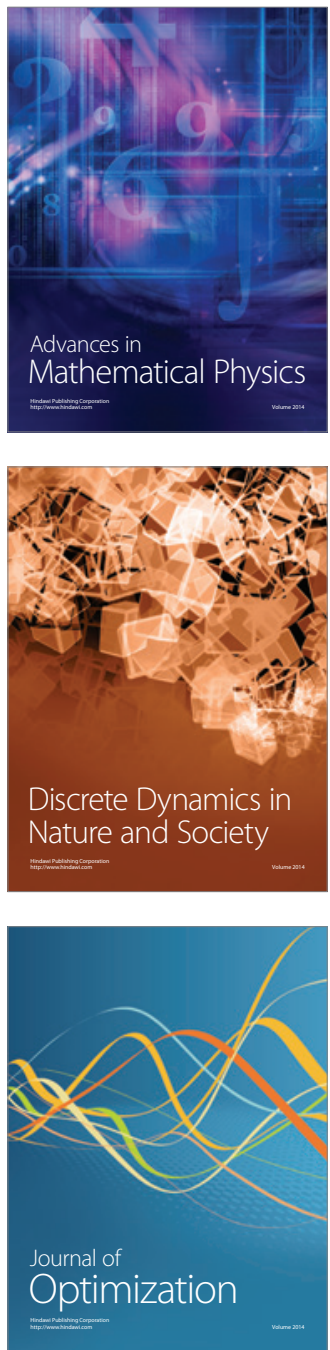